\author{
Comportamento Assintótico \\ de Estimadores da Entropia \\ para Cadeias de Ordem Infinita \\ com Perda de Memória Exponencial \\ Daniela Guiol
}

\title{
TESE APRESENTADA
}

$\mathrm{AO}$

INSTITUTO DE MATEMÁTICA E ESTATÍSTICA

DA UNIVERSIDADE DE SÃO PAULO

PARA OBTENÇÃO DO GRAU DE DOUTOR

EM

ESTATÍSTICA

Área de concentração: Teoria das Probabilidades

Orientador: Antonio Galves

Co-orientadora: Nancy L. Garcia

São Paulo, 15 de janeiro de 2002 


\section{Resumo}

Nesta tese, apresentaremos resultados sobre as flutuações de dois estimadores da entropia para uma classe de cadeias de ordem infinita.

Consideraremos uma cadeia estacionária de ordem infinita isto é, na qual as probabilidades de transição dependem de todo o passado. Faremos a hipótese hahitual em teoria de informação que a cadeia assume valores no alfabeto finito. Suponhamos também que a memória do passado decresce com velocidade exponencial.

A partir de uma amostra finita da cadeia, de comprimento $n$, consideraremos a $k$-ésima distribuição empírica de um cilindro de comprimento $k$ como sendo a frequência relativa do cilindro na amostra. Nesta tese, será estudado caso em que esse comprimento $k$ é uma função crescente do comprimento da amostra, isto é $k:=k(n)$.

Consideraremos dois estimadores da entropia. O primeiro deles é a $k$-ésima entropia empírica. Ele é definido como a razão entre a esperança, com respeito a distribuição empírica do logaritmo da probabilidade dos $k$-cilí ndros e o próprio comprimento $k$.

O segundo deles é a $k$-ésima entropia empírica condicionada. Ele é definido considerando-se a esperança, com respeito a distribuição empírica do logaritmo da probabilidade condicionada empírica.

No Capítulo 4 se encontram as contribuições originais desta tese. Os resultados principais são os Teoremas 4.6.4 e 4.6.5. Demonstramos no Teorema 4.6.4, que a $k$-ésima entropia empírica condicionada tem flutuações gaussianas em tomo) da verdadeira entropia do processo. Demonstramos também, no Teorema 4.6.5, que o mesmo não acontece com a entropia empírica de ordem $k$. Nossos resultados valem se $k(n)<\frac{1}{2 \log |\mathcal{A}|} \log n$. Esta é uma condição natural, já que $\log |\mathcal{A}|$ é majorante da entropia do processo e que, em um contexto de aplicação concreta, a entropia do processon não é conhecida a priori..

Os Teroremas 4.6 .4 e 4.6 .5 respondem a uma questão deixada em aberto desde () artigo de Iosifescu (1965). Em particular o Teorema 4.6 .5 aponta para uma diferença crucial entre cadeias de Markov e cadeias de ordem infinita. 


\begin{abstract}
This dissertation addresses the question of the fluctuations of two estimators of the entropy for a chain of infinite order. We assume that the chain takes values on a finite alphabet and loses memory exponentially fast. We prove a central limit theorem for the conditional entropy of the empirical distribution of the chain, for cylinders growing logarithmically with the length of the sample. We also prove that the entropy of the empirical distribution of the cylinders, divided by the length of the cylinders does not have Gaussian fluctuations.
\end{abstract}




\section{Sumário}

1 Introdução 3

2 Cadeias de ordem infinita $\quad 7$

2.1 Notações e definições . . . . . . . . . . . . . . . . . . 7

2.2 Uma construção regenerativa para cadeias de ordem infinita . . . . 9

2.2.1 Existência e unicidade de uma cadeia de ordem infinita . . 10

2.2.2 Regeneração. Resultados prévios . . . . . . . . . . . . 11

2.3 Resultados de Bressaud, Fernández e Galves (1999) . . . . . . . . 14

2.3.1 A aproximação canônica de Markov para cadeias de ordem infinita . . . . . . . . . . . . . . . . . . . 14

2.3.2 Velocidade de decaimento das correlações . . . . . . . . 15

3 Entropia de um processo estocástico 17

3.1 Definições e interpretação . . . . . . . . . . . . . . . . . . . 17

3.2 Estimadores da entropia . . . . . . . . . . . . . . . . . . . 20

3.3 Resultados prévios . . . . . . . . . . . . . . . . . . 21

4 Resultados novos. Flutuações da entropia empírica em torno da entropia do processo. $\quad 25$

4.1 Teorema-limite central . . . . . . . . . . . . . . 26

4.2 Regeneração. Propriedades . . . . . . . . . . . . . . . . . . . . 29

4.3 Covariâncias . . . . . . . . . . . . . . . . . . . . . . . 38

4.4 Prova do Teorema-limite central . . . . . . . . . . . . . . 46

4.5 Entropia relativa . . . . . . . . . . . . . . . . 58

4.6 Teorema-limite central para a entropia empírica . . . . . . . . . . . 59

4.6.1 Velocidade de convergência . . . . . . . . . . . 61

4.6.2 Flutuações dos dois estimadores da entropia em torno da entropia do processo . . . . . . . . . . . . 62 


\section{Capítulo 1}

\section{Introdução}

A entropia de um processo estocástico se revelou um conceito fundamental em diversas áreas científicas começando com a termodinâmica, a mecânica estatística, a teoria ergódica e mais recentemente a teoria da informação.

Do ponto de visto aplicado, em áreas como a teoria da informação uma questão central é a estimação da entropia de um processo a partir de uma longa porém finita realização do mesmo. Isso conduz naturalmente à questão da definição de estimadores para entropia e ao estudo de suas propriedades assintóticas.

Nesta tese, apresentaremos resultados sobre as flutuações de dois estimadores da entropia para uma classe de cadeias de ordem infinita.

Consideraremos uma cadeia estacionária de ordem infinita isto é, na qual as probabilidades de transição dependem de todo o passado. Faremos a hipótese habitual em teoria de informação que a cadeia assume valores no alfabeto finito. Suponhamos também que a memória do passado decresce com velocidade exponencial.

A partir de uma amostra finita da cadeia, de comprimento $n$, consideraremos a $k$-ésima distribuição empírica de um cilindro de comprimento $k$ como sendo a frequência relativa do cilindro na amostra. Nesta tese, será estudado caso em que esse comprimento $k$ é uma função crescente do comprimento da amostra, isto é $k=k(n)$.

Consideraremos dois estimadores da entropia. O primeiro deles é a $k$-ésima entropia empírica. Ele é definido como a razão entre a esperança, com respeito a distribuição empírica do logaritmo da probabilidade dos $k$-cilí ndros e o próprio comprimento $k$.

O segundo deles é a $k$-ésima entropia empírica condicionada. Ele é definido considerando-se a esperança, com respeito a distribuição empírica do logaritmo 
da probabilidade condicionada empírica.

Em relação aos dois estimadores se coloca a questão da consistência dos mesmos e o estudo de suas flutuações em torno do valor limite.

No caso quando o comprimento $k$ dos cilí ndros não depender do comprimento da amostra, como consequência da teoria ergódica clássica, temos os seguintes resultados. Quando o comprimento da amostra cresce , a $k$-ésima distribuição empírica converge quase certamente para a $k$-ésima distribuição verdadeira e temos também a convergência da $k$-ésima entropia empírica para a $k$-ésima entropia verdadeira. Segue que no caso de cadeias de Markov, isto é, de cadeias de ordem finita, a $k$ - ésima entropia condicionada coincide com a entropia do processo sempre que o $k$ for maior ou igual a ordem da cadeia. Neste caso os resultados clássicos acima mencionados mostram que os dois estimadores da entropia são consistentes.

No caso de cadeias de ordem infinita com perda de memória somável restringindose aos cilíndros de comprimento $k$ fixo, Iosifescu (1965) provou que os dois estimadores da entropia tem flutuações gaussianas em torno da $k$-ésima entropia do processo. Ou seja no caso de cadeia de Markov de ordem maior ou igual a $k 0$ resultado de Iosifescu (1965) mostra que a entropia empírica flutua em torno da entropia verdadeira do processo.

No caso de cadeia de ordem infinita, no entanto, a teoria ergódica clássica não resolve o problema da estimação da entropia do processo. Este é o tema da presente tese.

Para cadeias de ordem infinita temos que considerar cil' indros de comprimento $k(n)$, crescendo junto com o comprimento $n$ da amostra.

Ornstein e Weiss (1990) provaram que a distância em $\bar{d}$ entre a $k(n)$-ésima distribuição empírica e a $k(n)$-ésima distribuição verdadeira converge para zero se $k(n)$ cresce logaritmicamente com $n$. No mesmo contexto, Shields (1990) mostrou que a distância variacional (que majora a distância $\bar{d}$ ) entre a distribuição empírica e a distribuição verdadeira converge para zero se $k(n)<\frac{1}{h} \log n$, onde $h$ designa a entropia do processo.

Csiszár e Shields (2000), estimam a velocidade de convergência da razão entre a $k(n)$-ésima distribuição empírica e a distribuição verdadeira.

Para cadeias de Markov, Csiszár (2000), usando a teoria dos martingais, estima a diferença entre a distribuição empírica condicionada e a distribuição verdadeira condicionada e prova um resultado sobre a velocidade de convergência da entropia relativa.

Ornstein e Weiss (1990), provaram um resultado do tipo Teorema de ShannonMcMillan-Breiman, para a $k$-ésima entropia empírica. Dai segue que se o com- 
primento dos cilíndros cresce com $n$, mas não muito rápido, precisamente, se $k(n) \leq \frac{1}{h} \log n$ então a $k$-ésima entropia empírica é um estimador consistênte da entropia do processo.

Shields (2001), mostrou a consistência do outro estimador da entropia, a $k$ ésima entropia empírica condicionada, se $k(n)<\frac{1}{h} \log n$. No caso de uma sequência de variáveis independentes e uniformemente distribuídas no alfabeto de entropia $h=1$, Shields (2001), mostra que a entropia verdadeira não é atingida pela a $k(n)$-ésima entropia empírica condicionada se $k=\log n$.

Apresentaremos estes resultados sobre a consistênia dos estimadores da entropia no Capítulo 3.

No Capítulo 4 se encontram as contribuições originais desta tese. Os resultados principais são os Teoremas 4.6.4 e 4.6.5. Demonstramos no Teorema 4.6.4, que a $k$-ésima entropia empírica condicionada tem flutuações gaussianas em torno da verdadeira entropia do processo. Demonstramos também, no Teorema 4.6.5, que o mesmo não acontece com a entropia empírica de ordem $k$. Nossos resultados valem se $k(n)<\frac{1}{2 \log |\mathcal{A}|} \log n$. Esta é uma condição natural, já que $\log |\mathcal{A}|$ é majorante da entropia do processo e que, em um contexto de aplicação concreta, a entropia do processo não é conhecida a priori..

Os Teoremas 4.6 .4 e 4.6 .5 respondem a uma questão deixada em aberto desde o artigo de Iosifescu (1965). Em particular o Teorema 4.6.5 aponta para uma diferença crucial entre cadeias de Markov e cadeias de ordem infinita.

A prova destes teoremas é baseada em dois resultados originais nossos, interessantes por si só. O primeiro resultado, o Teorema 4.1.1, é um teorema-limite central para cadeias de ordem infinita observadas atraves de uma função cilíndrica de comprimento $k(n)$. O segundo resultado, o Teorema 4.5.1, é sobre a convergência da entropia relativa da distribuição empírica com respeito a distribuição verdadeira.

A prova do Teorema 4.1.1 é baseada na construção regenerativa de cadeias de ordem infinita , apresentada em Comets, Fernández e Ferrari (2000). Com essa construção, o processo pode ser visto como uma sequência de vetores aleatórios independêntes. Para concluir a demonstração usamos o Teorema de Berry-Essen, que estima a velocidade de convergência para a distribuição normal, no caso, de variáveis aleatórias independentes e identicamente distribuídas..

A prova do Teorema 4.5.1 é baseada em resultados de Bressaud, Fernández e Galves (1999b), sobre a velocidade de decaimento de correlações de cadeias de ordem infinita com perda de memória somável.

Concluímos esta introdução com uma breve recapitulação histórica. Cadeias de ordem infinita foram consideradas por vários autores, sob diversas designações. 
Onicescu e Mihoc (1935) são iniciadores da pesquisa na área logo seguidos por Doeblin e Fortet (1937). As primeiras demonstrações de teoremas-limite centrais para funções cilíndricas de comprimento fixo se encontram em Onicescu e Mihoc (1940), Iosifescu (1961), Mihoc (1963). Harris (1955) introduziu a idéia de construção regenerativa para cadeias de ordem infinita. Esquemas regenerativas reaparecem em Athreya e Ney (1978), Nummelin (1978), Lalley (1986) e Berbee (1987) e, mais recentemente, em Ferrari, Martinez e Ney (2000) e Comets, Fernández e Ferrari (2000).

O conceito de taxa de entropia de um processo estocástico foi introduzido em Shannon (1948), que explorou algumas conexões entre a taxa de entropia de um processo estocástico e o número de possíveis sequências geradas pelo processo. Os trabalhos de Shannon marcam o início da teoria da informação.

Uma apresentação histórica mais ampla das cadeias de ordem infinita pode ser encontrada no livro de Iosifescu e Grigorescu (1990). Uma apresentação moderna da teoria se encontra em Fernández, Ferrari e Galves (2001). Questões relativas a entropia no quadro da teoria ergódica e teoria da informação se encontra em Cover e Thomas (1991), Gray(1990), e Shields (1996). 


\section{Capítulo 2}

\section{Cadeias de ordem infinita}

\subsection{Notações e definições}

Nesta seção definiremos o conceito de cadeia de ordem infinita, positiva e logcontínua. Em Lalley (1986) essa classe particular de cadeias de ordem infinita é chamada de cadeia com conexões completas.

Consideraremos $\mathcal{A}$ um conjunto finito que chamaremos alfabeto. Denotaremos com $|\mathcal{A}|$ o número de elementos do alfabeto e $\mathcal{A}^{\mathbb{Z}}$ o espaço das sequências infinitas

$$
x=\left(x_{i}\right), x_{i} \in \mathcal{A}, i \in \mathbb{Z} .
$$

Para $-\infty \leq m \leq n \leq+\infty$ denotaremos $x_{m}^{n}$ a sequência $x_{m}, . ., x_{n}$.

Denotamos $\operatorname{com} T: \mathcal{A}^{\mathbb{Z}} \rightarrow \mathcal{A}^{\mathbb{Z}}$ o operador de translação à esquerda em uma coordenada, isto é:

$$
(T(x))_{n}=x_{n+1}, x \in \mathcal{A}^{\mathbb{Z}}, n \in \mathbb{Z} .
$$

Definição 2.1.1. Chamaremos de probabilidade de transição uma função

$$
P(\cdot \mid \cdot): \mathcal{A} \times \mathcal{A}^{-\mathbb{N}^{*}} \rightarrow[0,1]
$$

que satisfaz as seguintes condições:

(i) Mensurabilidade: Para cada $x_{0} \in \mathcal{A}$ a função $P\left(x_{0} \mid.\right)$ é mensurável com respeito à $\sigma$-álgebra produto.

(ii) Normalização: Para cada $x_{-\infty}^{0} \in \mathcal{A}^{-\mathbb{N}}$ vale

$$
\sum_{x_{0} \in \mathcal{A}} P\left(x_{0} \mid x_{-\infty}^{-1}\right)=1
$$


Consideraremos um processo estocástico estacionário, $\left(X_{n}\right)_{n \in Z}$, definido sobre um espaço de probabilidade $(\Omega, \Sigma, \mathbf{P})$ com valores no alfabeto $\mathcal{A}$.

Definição 2.1.2. Se existe $k \geq 1$ tal que para qualquer $a_{-\infty}^{0} \in \mathcal{A}^{-\mathbb{N}}$ vale

$$
\mathbf{P}\left(X_{0}=a_{0} \mid X_{-k-m}^{-1}=a_{-k-m}^{-1}\right)=\mathbf{P}\left(X_{0}=a_{0} \mid X_{-k}^{-1}=a_{-k}^{-1}\right) .
$$

para todo $m \geq 1$ então chamaremos $\left(X_{n}\right)_{n \in Z}$ de cadeia de Markov de ordem $k$.

Se não existe nenhum $k$ satisfazendo (2.1.1), dizemos que a cadeia é de ordem infinita (c.o.i.).

Consideraremos $P(\cdot \mid \cdot)$ uma probabilidade de transição definida sobre o espaço $\mathcal{A} \times \mathcal{A}^{-\mathbb{N}^{*}}$.

Definição 2.1.3. Dizemos que $\left(X_{n}\right)_{n \in Z}$ é consistente com a probabilidade de transição P se para cada $x \in \mathcal{A}^{-\mathbb{N}}$ vale

$$
\mathbf{P}\left(X=x_{0} \mid X_{-\infty}^{-1}=x_{-\infty}^{-1}\right)=P\left(x_{0} \mid x_{-\infty}^{-1}\right) .
$$

Para sequências $x, y \in \mathcal{A}^{-\mathbb{N}}$ denotaremos por $x \stackrel{k}{=} y$ sempre que $x_{-k}^{0}=y_{-k}^{0}$.

Definição 2.1.4. Chamaremos de cadeia de ordem infinita positiva e log-contínua (c.o.i.p.l.c.) com taxa de perda de memória $\left(\gamma_{k}\right)_{k \geq 1}$, uma cadeia de ordem infinita, $\left(X_{n}\right)_{n \geq 1}$, consistente com a probabilidade de transição P que satisfaz as seguintes condições:

(i) positividade:

$$
\inf _{x \in \mathcal{A}^{-\mathrm{N}}} P\left(x_{0} \mid x_{-\infty}^{-1}\right)>0
$$

(ii) log-continuidade:

$$
\lim _{k \rightarrow \infty} \gamma_{k}=0
$$

onde

$$
\gamma_{k}:=\sup _{\{x, y: x=y\}}\left|\frac{P\left(x_{0} \mid x_{-\infty}^{-1}\right)}{P\left(y_{0} \mid y_{-\infty}^{-1}\right)}-1\right| .
$$

Se a taxa de perda de memória decresce exponencialmente, isto é, existe um $c>0$ tal que $\gamma_{k}=\exp (-c k)$, chamaremos $\left(X_{n}\right)_{n \geq 1}$ de cadeia de ordem infinita positiva e log-contínua com perda de memória exponencial. (c.o.i.p.l.c.e.). 
Definição 2.1.5. Chamaremos de aproximação canônica de Markov de ordem $k \geq 1$ de um processo $\left(X_{n}\right)_{n \in Z}$, uma cadeia de Markov de ordem $k$ cuja a probabilidade de transição $P^{k}$ satisfaz

$$
P^{k}\left(a_{0} \mid a_{-k}^{-1}\right)=\mathbf{P}\left(X_{0}=a_{0} \mid X_{-k}^{-1}=a_{-k}^{-1}\right)
$$

para qualquer $a_{-k}^{0} \in \mathcal{A}^{k+1}$.

Observação 2.1.6. Apresentaremos, na Seção 2.3, um resultado do Bressaud, Fernández e Galves (1999a), que compara as probabilidades de transição de $\left(X_{n}\right)_{n \in Z}$, uma c.o.i.p.l.c. com taxa de perda de memória somável e da aproximação canônica de Markov de ordem $k \geq 1$ correspondente.

Seja $n>m$. Chamaremos de $(n-m)$ - cilíndro, definido a partir da sequência $a_{m}^{n}$, o seguinte subconjunto de $\mathcal{A}^{\mathbb{Z}}$ :

$$
\left[a_{m}^{n}\right]:=\left\{x \in \mathcal{A}^{\mathbb{Z}}: x_{m}^{n}=a_{m}^{n}\right\}
$$

Denotaremos por $\mathcal{F}_{n}$ o conjunto de todos os n-cilindros. Denotaremos também por $\mathcal{F}$ a $\sigma$ - álgebra definida sobre $\mathcal{A}^{Z}$ gerada pelos cilíndros.

Denotaremos por $\nu$ a única medida de probabilidade sobre $\mathcal{A}^{\mathbb{Z}}$, invariante por translações tal que para cada $n \in \mathbb{Z}$ e para cada $a_{1}^{n} \in \mathcal{A}^{n}$

$$
\nu\left(\left[a_{1}^{n}\right]\right):=\mathbf{P}\left(X_{1}^{n}=a_{1}^{n}\right) .
$$

Consideraremos $\nu$ a medida invariante de um processo estacionário $(X)_{n \in \mathbb{Z}}$. Para conveniência usaremos as seguintes notações: $\nu\left(a_{m}^{n}\right)=\nu\left(\left[a_{m}^{n}\right]\right)$.

Denotaremos as probabilidades condicionais como:

$$
\nu\left(a_{k}^{l} \mid a_{m}^{n}\right)=\frac{\nu\left(\left[a_{k}^{l}\right] \cap\left[a_{m}^{n}\right]\right)}{\nu\left(\left[a_{m}^{n}\right]\right)} .
$$

\subsection{Uma construção regenerativa para cadeias de ordem infinita}

Nesta seção apresentaremos a construção regenerativa de Comets, Fernández e Ferrari (2000), para cadeias de ordem infinita com perda de memória somável. Este esquema regenerativo será a base da prova do teorema-limite central para cadeias de ordem infinita que provaremos no Capítulo 4. Consideraremos $U=$ 
$\left(U_{i}\right)_{i \in \mathbb{Z}}$ uma sequência de variáveis aleatórias independentes e uniformemente distribuídas em $[0,1)$, construídas sobre o espaço de probabilidade $(\Omega, \Sigma, \mathbf{P})$, onde $\Sigma$ é a $\sigma$-álgebra gerada pela sequência de variáveis aleatórias uniformes $U$.

Consideraremos $\left(X_{n}\right)_{n \geq 1}$ uma c.o.i. de probabilidade de transição $P$ e definida sobre o espaço de probabilidade $(\Omega, \Sigma, \mathbf{P})$.

\subsubsection{Existência e unicidade de uma cadeia de ordem infinita}

De fato, em Comets, Fernández e Ferrari (2000), é apresentada uma construção explícita, de uma c.o.i. compatível com a probabilidade de transição $P$, a partir da sequência de variáveis aleatórias uniformes $U$. Como consequência desta construção segue o teorema de existência e unicidade apresentada nesta Seção.

Definição 2.2.1. Seja $P$ uma probabilidade de transição. Para cada $k \in \mathbb{N}, x_{0} \in$ $\mathcal{A}$ e $x_{-k}^{-1} \in \mathcal{A}^{k}$ definiremos as seguintes funções:

$$
\begin{array}{r}
\alpha_{0}\left(x_{0}\right):=\inf _{x_{-\infty}^{-1}} P\left(x_{0} \mid x_{-\infty}^{-1}\right) ; \\
\alpha_{k}\left(x_{0} \mid x_{-k}^{-1}\right):=\inf _{z_{-\infty}^{-k-1}} P\left(x_{0} \mid x_{-k}^{-1} z_{-\infty}^{-k-1}\right) . \\
\alpha_{k}:=\min _{x_{-k}^{-1} \in \mathcal{A}^{k}}\left(\sum_{x_{0} \in \mathcal{A}} \alpha_{k}\left(x_{0} \mid x_{-k}^{-1}\right)\right) . \\
\beta_{m}:=\prod_{k=0}^{m} \alpha_{k}
\end{array}
$$

onde $\left(x_{-k}^{-1} z_{-\infty}^{-k-1}\right)=\left(\ldots, z_{-k-1}, x_{-1}, . ., x_{-k}\right)$.

Definição 2.2.2. Chamaremos de tempo de regeneração de uma janela $[l, m]$, para $-\infty<l<\infty$, el $\leq m \leq \infty$ á variável aleatória

$$
\tau[l, m]:=\max \left\{t \leq l: U_{n}<\alpha_{n-t}, n \in[l, m]\right\} .
$$

Por convenção $\tau[l, m]=-\infty$ se o conjunto do lado direito é vazio.

Se $l=m$, escrevemos $\tau[l]:=\tau[l, l]$.

Teorema 2.2.3. Se P for uma probabilidade de transição tal que

$$
\sum_{m \geq 0} \prod_{k=0}^{m} \alpha_{k}=\infty
$$


então valem as seguintes propriedades:

(i) para cada janela finita $[l, m]$

$$
\mathrm{P}(\tau[l, m]>-\infty)=1 .
$$

(ii) Existe um único processo estocástico estacionário $\left(X_{n}\right)_{n \in \mathbb{Z}}$, definido sobre o espaço $(\Omega, \Sigma, \mathrm{P})$, compatível com $P$.

(iii) Existe uma função $\Phi:[0,1)^{\mathbb{Z}} \rightarrow \mathcal{A}^{Z}$ mensurável tal que $X_{n}=\Phi(U)(n)$, para cada $n \in \mathbb{Z}$.

Para cada intervalo finito $[l, m]$ a restrição $\{\Phi(U)(i): i \in[l, m]\}$ depende somente de $\left\{U_{i}: i \in[\tau[l, m], m]\right\}$.

\subsubsection{Regeneração. Resultados prévios}

Nesta subseção apresentaremos um teorema, provado em Comets, Fernández e Ferrari (2000), que mostra que a construção apresentada na subseção precedente, é realizada de maneira regenerativa.

De fato, a partir da sequência de variáveis aleatórias uniformes $U$, são construídos em mesmo tempo, uma c.o.i compatível com a probabilidade de transição $P$ e por outro lado um processo de renovação $\left(\tau_{i}\right)_{i \in \mathbb{Z}}$ de tal jeito que a c.o.i. $\left(X_{n}\right)_{n \geq 1}$ pode ser vista como uma sequência de vetores aleatórios $X_{\tau_{i}}^{\tau_{i+1}-1}$, de comprimento aleatório $\tau_{i+1}-\tau_{i}$, independentes e identicamente distribuídos.

Observação 2.2.4. Na Definição 2.2.1, para cada l fixo, a sequência dos tempos de regeneração $\tau[l, m]$ para $m \geq l$ é decrescente, portanto existe o limite

$$
\tau[l,+\infty):=\lim _{m \rightarrow+\infty} \tau[l, m] .
$$

Definição 2.2.5. Se $\tau[l,+\infty)>-\infty$ chamaremos as variáveis $\tau[l,+\infty)$ de tempos de regeneração do processo.

Teorema 2.2.6. Se P é uma probabilidade de transição tal que

$$
\prod_{k=0}^{\infty} \alpha_{k}>0 .
$$

então valem as seguintes propriedades

(i) Para cada $l \in \mathbb{Z}$ temos $\mathbf{P}(\tau[l, \infty)>-\infty)=1$.

(ii) Existe um único processo estocástico estacionário $\left(X_{n}\right)_{n \in \mathbb{Z}}$ compatível com $P$. 
(iii) Existe uma função $\Phi:[0,1)^{\mathbb{Z}} \rightarrow \mathcal{A}^{Z}$ mensurável tal que $X_{n}:=\Phi(U)(n)$, para cada $n \in \mathbb{Z}$.

Para cada intervalo infinito $[l,+\infty)$ a restrição $\{\Phi(U)(i): i \in[l,+\infty)\}$ depende somente de $\left(U_{i}\right)_{i \geq \tau[l,+\infty)}$.

Consideraremos as variáveis aleatórias $W \in\{0,1\}^{\mathbb{Z}}$ definidas por

$$
W_{j}:=1\{\tau[j,+\infty)=j\} .
$$

Sejam $\left(\tau_{l}\right)_{l \in \mathbb{Z}}$, os tempos ordenados correspondentes as realizações de $W$, definidos por

$$
\begin{aligned}
\tau_{-1}: & =\sup \left\{j \leq 0: W_{j}=1\right\} \\
\tau_{i}: & =\inf \left\{j>\tau_{i-1}: W_{j}=1\right\} \text { para } i \geq 0 \\
\tau_{-i}: & =\sup \left\{j<\tau_{-i+1}: W_{j}=1\right\} \text { para } i \geq 2
\end{aligned}
$$

Denotaremos $\triangle_{\tau_{i}}:=\tau_{i+1}-\tau_{i}$.

Observação 2.2.7. Note que $\tau_{l}$ é o l-ésimo tempo de regeneração do processo tal que $\tau[l,+\infty)=l$ que é diferente de $\tau[l]$, o tempo de regeneração da janela $[l, l]$.

Definição 2.2.8. Chamaremos de cadeia do castelo de cartas, uma cadeia de Markov $\left(Z_{n}\right)_{n \geq 0}$, com valores em $\mathbb{N}$, começando no tempo zero na origem e tal que a probabilidade de transição $P$ satisfaz

$$
P(y \mid x)= \begin{cases}\alpha_{x}, & y=x+1 \\ 1-\alpha_{x}, & y=0 \\ 0, & \text { caso contrário }\end{cases}
$$

Definiremos $\gamma_{n}^{*}$ como sendo a probabilidade do evento: cadeia do castelo de cartas retorna a zero no tempo $n$. Isto é,

$$
\gamma_{n}^{*}:=\mathbf{P}\left(Z_{n}=0\right)
$$

Lema 2.2.9. Sejam $\left(\alpha_{k}\right)_{k \geq 1}$ uma sequência crescente, $\alpha_{k} \nearrow 1$, de reais positivos $e\left(\gamma_{n}^{*}\right)_{n \in \mathbb{Z}}$ a sequêcia definida em (2.2.5). Então

1) $\sum_{n \geq 1} \prod_{k=0}^{n} \alpha_{k}=+\infty$ se e somente se $\gamma_{n}^{*} \rightarrow 0$.

2) $\prod_{k=0}^{+\infty} \alpha_{k}>0$ se e somente se $\sum_{n \geq 0} \gamma_{n}^{*}<+\infty$.

3) Se $\left(1-\alpha_{k}\right)_{k \geq 0}$ decresce exponencialmente então $\left(\gamma_{n}^{*}\right)_{n \geq 0}$ também decresce exponencialmente. 
Denotaremos

$$
\beta:=\prod_{k=0}^{+\infty} \alpha_{k} .
$$

Teorema 2.2.10. Se P for uma probabilidade de transição tal que $\beta>0$ então valem

(i) O processo $\left(W_{j}\right)_{j \geq 0}$ é um processo de renovação estacionário com $\mathrm{P}\left(W_{j}=\right.$ 1) $=\beta e$

$$
\mathbf{P}\left(\tau_{l+1}-\tau_{l} \geq m\right)=\gamma_{m}^{*}
$$

para $m \geq 1$ el $l \neq 0$.

(ii) Os vetores aleatórios $\xi_{l} \in \cup_{n \geq 1} \mathcal{A}^{n}, l \in \mathbb{Z}$ definidos por $\xi_{l}=\left(X_{\tau_{l}}, . ., X_{\tau_{l+1}-1}\right)$ são independentes e identicamente distribuídos.

Como consequência do Teorema 2.2.10 e do Lema 2.2.9 temos o seguinte resultado que mostra que para c.o.i.p.l.c. com perda de memória exponencial vale a construção regenerativa e, para todo $i \geq 1$, os incrementos $\Delta \tau_{i}$ tem momentos finitos de qualquer ordem. Este resultado será útil para provar o teorema-limite central do Capítulo 4.

Corolario 2.2.11. Se $\left(X_{n}\right)_{n \in \mathbb{Z}}$ é uma c.o.i.p.l.c.e. então

i) $\beta>0$.

ii) a sequência $\gamma_{m}^{*}$ decresce exponencialmente.

iii) Para todo $i \geq 1$ as variáveis aleatórias $\Delta_{\tau_{i}}$, são i.i.d. e tais que a distribuição comum é majorada por uma distribuição geométrica. Então para todo $m \geq 1$ temos

$$
\mathrm{E}\left(\left|\Delta_{\tau_{1}}\right|^{m}\right)<+\infty
$$

iv) Os vetores $\left(X_{\tau_{i}}, \ldots, X_{\tau_{i+1}-1}\right), i \in \mathbb{Z}^{*}$ são i.i.d.

Prova. Segue da log-continuidade da probabilidade de transição $P$ que para cada $a_{0} \in \mathcal{A}$ e $v \in \mathcal{A}^{-\mathbb{N}^{*}}$

$$
\inf \left\{\mathbf{P}\left(a_{0} \mid x_{-1}, \ldots, x_{-k} z\right): z \in \mathcal{A}^{-\mathbb{N}^{*}}\right\} \geq\left(1-\gamma_{k}\right) \mathbf{P}\left(a_{0} \mid x_{-1}, \ldots, x_{-k} v\right) .
$$

Somando segundo $a_{0} \in \mathcal{A}$ e tomando o minimo sobre $x_{-1}^{-k}$ concluímos que

$$
\alpha_{k} \geq 1-\gamma_{k}
$$


Como $\left(\gamma_{k}\right)_{k \geq 0}$ decresce exponencialmente então a soma $\sum_{k \geq 0}\left(1-\alpha_{k}\right)<+\infty$. Isto implica que $\prod_{k=0}^{+\infty} \alpha_{k}>0$. Como consequência do Lema 2.2.9 temos $\gamma_{m}^{*}=$ $\left(\gamma_{1}^{*}\right)^{m}$ para todo $m \geq 1$. Usando o Teorema 2.2.10 temos

$$
\mathbf{P}\left(\Delta_{\tau_{1}}=m\right)<\left(\gamma_{1}^{*}\right)^{m-1}\left(1-\gamma_{1}^{*}\right)
$$

Isto conclui a prova do corolário.

Denotaremos por

$$
\gamma:=\mathbf{E}\left(\Delta_{\tau_{1}}\right)
$$

\subsection{Resultados de Bressaud, Fernández e Galves (1999)}

\subsubsection{A aproximação canônica de Markov para cadeias de or- dem infinita}

O Lema que apresentaremos nesta subseção foi provado em Bressaud, Fernández e Galves (1999a).

Lema 2.3.1. Sejam $\left(X_{n}\right)_{n \in Z}$ uma c.o.i.p.l.c. com taxa de perda de memória $\left(\gamma_{k}\right)_{k \geq 1}$ e $P^{k}$ a probabilidade de transição da aproximação canônica de Markov de ordem $k \in N$ do processo $\left(X_{n}\right)_{n \in Z}$. Para cada $k \geq 1$ valem as seguintes propriedades:

(i) Para cada $m \geq 1$ temos

$$
\sup \left\{\left|\frac{P\left(x_{0} \mid x_{-\infty}^{-1}\right)}{P^{k}\left(x_{0} \mid y_{-k}^{-1}\right)}-1\right|: x, y \in \mathcal{A}^{-\mathbb{N}}, x_{-m}^{-1}=y_{-m}^{-1}\right\} \leq \gamma_{\text {inf }\{m, k\}} .
$$

(ii)

$$
\inf _{\left\{x, y: x_{-\infty}^{0} \underline{\underline{k}} y_{-\infty}^{0}\right\}} P\left(x_{0} \mid x_{-\infty}^{-1}\right) \leq P^{k}\left(x_{0} \mid y_{-\infty}^{-1}\right) \leq \sup _{\left\{x, y: x_{-\infty}^{0} \underline{\underline{k}} y_{-\infty}^{0}\right\}} P\left(x_{0} \mid x_{-\infty}^{-1}\right) .
$$

Como consequência deste resultado provaremos que a entropia de uma c.o.i.p.l.c. é positiva não-nula. Usaremos também este Lema na Seção 4.3, para estudar as covariâncias de algumas funções cilíndricas particulares. 


\subsubsection{Velocidade de decaimento das correlações}

O teorema apresentado nesta seção foi provado em Bressaud, Fernández e Galves (1999b). Este teorema será, na Seção 4.3, uma ferramenta de base para estudar o comportamento assintótico das covariâncias.

Chamaremos de velocidade de decaimento das correlações de ordem $d \geq 0$, ou taxa de mistura, a velocidade de convergência do limite

$$
\mathrm{E}_{\nu}\left(f T_{-d} g\right) \stackrel{d \rightarrow \infty}{\rightarrow} \mathrm{E}_{\nu} f \mathbf{E}_{\nu} g
$$

Definição 2.3.2. Sejam $f, g \in L^{2}(d \nu)$. Chamaremos $C_{f, g}(d)$ as covariâncias de ordem $d \geq 0$ das funções $f$ e $g$

$$
C_{f, g}(d):=\int d \nu f T_{d} g-\left(\int d \nu f\right)\left(\int d \nu g\right)
$$

Se $f=g$, denotaremos com $C_{f}(d)$ a covariância $C_{f, f}(d)$.

Denotaremos

$$
\left(\sigma_{f}^{2}\right)^{m}:=\mathbf{E}_{\nu}\left[\left(\frac{1}{\sqrt{m}} \sum_{d=1}^{m}\left(T_{d} f-\mathbf{E}_{\nu} f\right)\right)^{2}\right] .
$$

Observamos que

$$
\left(\sigma_{f}^{2}\right)^{m}=C_{f}(0)+2 \sum_{d=1}^{m-1} C_{f}(d)-\frac{2}{m} \sum_{d=1}^{m-1} d C_{f}(d) .
$$

Se $\sum_{d=1}^{+\infty} d C_{f}(d)<+\infty$ então podemos definir

$$
\sigma_{f}^{2}:=\lim _{m \rightarrow \infty}\left(\sigma_{f}^{2}\right)^{m}=C_{f}(0)+2 \sum_{d=1}^{+\infty} C_{f}(d) .
$$

Consideraremos $f: \mathcal{A}^{-\mathbb{N}} \rightarrow \mathbb{R}$. Chamaremos $\operatorname{var}_{m}(f)$ de variação de ordem $m \geq 1$ de $f$, onde

$$
\operatorname{var}_{m}(f):=\sup _{\left\{x, y \in \mathcal{A}^{-\mathrm{N}}: x^{m}=y\right\}}|f(x)-f(y)| .
$$


Por conveniência denotaremos de

$$
\operatorname{var}_{-n}(f):=\sup _{\left\{x, y \in \mathcal{A}^{-\mathbb{N}}\right\}}|f(x)-f(y)| .
$$

para todo $n \geq 0$. Observamos que $\operatorname{var}_{-n}(f) \leq 2\|f\|_{\infty}$, para todo $n \geq 0$.

Algumas propriedades assintóticas das covariâncias e das variações serão provadas na Seção 4.3.

Definiremos a função $\phi: \mathcal{A}^{-\mathbb{N}} \rightarrow \mathbb{R}$

$$
\phi(x):=-\log \nu\left(x_{0} \mid x_{-\infty}^{-1}\right) .
$$

Denotaremos por $\mathcal{C}(\mathcal{A}, \mathbb{R})$ o espaço das funções reais contínuas definidas sobre $\mathcal{A}$.

Para cada $g \in \mathcal{C}(\mathcal{A}, \mathbb{R})$ definiremos a semi-norma

$$
\|g\|_{\phi}=\sup _{k \geq 0} \frac{\operatorname{var}_{k}(g)}{\operatorname{var}_{k}(\phi)}
$$

e denotaremos de $V_{\phi}=\left\{g \in \mathcal{C}(\mathcal{A}, \mathbb{R}):\|g\|_{\phi}<+\infty\right\}$.

\section{Teorema 2.3.3. (Bressaud, Fernández, Galves 1999b)}

Se $\left(X_{n}\right)_{n \in \mathbb{Z}}$ é uma c.o.i.p.l.c. com taxa de perda de memória somável e de medida invariante $\nu$, então para toda as funções $g \in V_{\phi}$ e $f \in L^{1}(\nu)$ e para todo $d \geq 0$ vale

$$
\left|C_{f, g}(d)\right| \leq C\|f\|_{1}\|g\|_{\phi} \gamma_{d}^{*}
$$

onde $\gamma_{n}^{*}$ são os tempos de retorno definidos em (2.2.5).

Usaremos este teorema na Seção 4.3.

Observação 2.3.4. Se a taxa de perda de memória do processo é exponencial então $\gamma_{d}^{*}$ também decresce exponencialmente e se as funções $f, g$ são limitadas temos que as covariâncias de ordem d decrescem exponencialmente em d. 


\section{Capítulo 3}

\section{Entropia de um processo estocástico}

Neste capítulo apresentaremos a definição e uma das possíveis interpretações clássicas da entropia de um processo estocástico. Definiremos dois estimadores da entropia e apresentaremos uma sequência de resultados sobre o comportamento assintótico destes estimadores.

\subsection{Definições e interpretação}

Definição 3.1.1. Seja p uma densidade de probabilidade sobre um alfabeto finito $\mathcal{A}$.

A entropia de $p$ é definida como

$$
H(p):=-\sum_{a \in \mathcal{A}} p(a) \log p(a)
$$

com a convenção $0 \log 0=0$. Consideraremos logaritmo em base 2.

Lema 3.1.2. A função entropia $H(p)$ é côncava e atinge seu valor máximo $\log |A|$ somente para a distribuição uniforme, $p(a)=\frac{1}{|A|}, a \in \mathcal{A}$.

A prova deste Lema pode ser encontrada em Cover e Thomas (1991).

Consideraremos $\left(X_{n}\right)_{n \in \mathbb{Z}}$ um processo estacionário e $\nu$ a medida invariante correspondente definida sobre o espaço $A^{\mathbb{Z}}$.

Apresentaremos o cé lebre Teorema de Shannon-McMillan-Breiman. Uma prova, mais recente deste Teorema encontra-se em Ornstein e Weiss (1983).

\section{Teorema 3.1.3. (Shannon-McMillan-Breiman)}


Se $\nu$ é uma medida ergódica então existe um número positivo $h=h(\nu)$ tal que $\nu$-quase certamente vale

$$
-\lim _{n \rightarrow \infty} \frac{1}{n} \log \nu\left(x_{1}^{n}\right)=h .
$$

Observação 3.1.4. De fato, o Teorema 3.1.3 mostra que para processos ergódicos $\nu\left(X_{1}^{n}\right)$ decresce quase-certamente exponencialmente em $n$ com uma taxa constante h chamada de taxa de entropia do processo.

Definição 3.1.5. Sejam $\varepsilon>0$ e $n \geq 1$. Chamaremos $T_{n}(\varepsilon)$ de conjunto das sequências típicas de comprimento $n$, o conjunto

$$
T_{n}(\varepsilon):=\left\{x_{1}^{n}: 2^{-n(h+\varepsilon)} \leq \nu\left(x_{1}^{n}\right) \leq 2^{-n(h-\varepsilon)}\right\} .
$$

Como consequência do Teorema 3.1.3 temos o seguinte resultado, que é uma expressão do teorema precedente numa forma exponencial e que introduz o conceito de conjunto de sequências típicas.

Teorema 3.1.6. Seja $T_{n}(\epsilon)$ o conjunto das sequências típicas de comprimento $n$ definido em (3.1.2).

1) $S e x_{1}^{n} \in T_{n}(\varepsilon)$ então

$$
h(\nu)-\varepsilon \leq-\frac{1}{n} \log \nu\left(x_{1}^{n}\right) \leq h(\nu)+\varepsilon .
$$

2)

$$
\lim _{n \rightarrow \infty} \nu\left(T_{n}(\varepsilon)\right)=1
$$

3)

$$
(1-\varepsilon) 2^{n(h-\varepsilon)} \leq\left|T_{n}(\varepsilon)\right| \leq 2^{n(h+\varepsilon)} .
$$

Interpretação: Como consequência do Teorema 3.1.3, sabemos que existe um conjunto $T_{n}(\varepsilon)$ que tem as seguintes propriedades: para $n$ suficientemente grande a medida deste conjunto é próxima de 1 , todos os elementos deste conjunto são quase equiprováveis e o número das sequências típicas de comprimento $n$ é próximo de $2^{\text {nh }}$.

Seja um processo estacionário de medida invariante $\nu$. Para cada $k \geq 1$ denotaremos por $\nu_{k}$ os marginais de ordem $k$ da medida $\nu$. Consideraremos as seguintes funções: $\phi_{k}, e_{k}: A^{k+1} \rightarrow \mathbb{R}$ tal que

$$
\begin{aligned}
& \phi_{k}\left(x_{-k}^{0}\right):=-\log \nu\left(x_{0} \mid x_{-k}^{-1}\right) ; \\
& e_{k}\left(x_{-k}^{0}\right):=-\log \nu\left(x_{-k}^{0}\right) .
\end{aligned}
$$

Algumas propriedades dessas funções serão provadas na Seção 4.3. 
Definição 3.1.7. 1) Chamaremos $\frac{1}{k} H\left(\nu_{k}\right)$ de k-ésima entropia do processo onde

$$
\begin{aligned}
H\left(\nu_{k}\right) & :=-\sum_{a_{1}^{k} \in A^{k}} \nu_{k}\left(a_{1}^{k}\right) \log \nu_{k}\left(a_{1}^{k}\right) \\
& =-k \mathbb{E}\left(e_{k}\right)
\end{aligned}
$$

2) Chamaremos $h\left(\nu_{k+1}\right)$ de k-ésima entropia condicionada do processo onde

$$
\begin{aligned}
h\left(\nu_{k+1}\right) & :=-\sum_{a_{-k}^{0} \in A^{k+1}} \nu_{k+1}\left(a_{-k}^{0}\right) \log \nu_{k+1}\left(a_{0} \mid a_{-k}^{-1}\right) . \\
& =-\mathbb{E}\left(\phi_{k}\right)
\end{aligned}
$$

Por conveniência denotaremos ás vezes $H\left(\nu_{k}\right):=H_{k}$ e $h\left(\nu_{k+1}\right):=h_{k}$.

Teorema 3.1.8. Seja $\left(X_{n}\right)_{n \in Z}$ um processo estacionário de medida invariante $\nu$ e $h(\nu)$ a taxa da entropia do processo definida em (3.1.1). Valem as seguintes propriedades:

1)

$$
H(\nu):=\lim _{k \rightarrow \infty} \frac{1}{k} H\left(\nu_{k}\right)
$$

$e$

$$
H(\nu)=\lim _{k \rightarrow \infty} h\left(\nu_{k+1}\right) .
$$

2) $\mathrm{Se}\left(X_{n}\right)_{n \in Z}$ é um processo estacionário ergódico então $H(\nu)=h(\nu)$.

Este Teorema é provado em Shields (1996).

Definição 3.1.9. Seja $X=\left(X_{i}\right)_{i \in Z}$ um processo estacionário ergódico de medida $\nu$. Chamaremos $h(\nu)$, definida em (3.1.1), (3.1.4) e (3.1.5), de entropia do processo $X$.

Observação 3.1.10. Observamos em general, que para calcular a entropia de um processo estacionário temos que achar o limite da k-ésima entropia do processo ou da k-ésima entropia condicionada do processo quando k cresce. Se X é uma cadeia de Markov estacionária temos uma fórmula explícita exata para calcular a entropia do processo. Isto é, para qualquer $k \geq 1$, temos $h\left(\nu_{k+1}\right)=h\left(\nu_{2}\right)=$ $h(\nu)$.

Teorema 3.1.11. Se $X=\left(X_{n}\right)_{n \in Z}$ é uma cadeia de Markov estacionária de medida invariante $\pi$ e de probabilidade de transição $P$ então

$$
h(\nu)=-\sum_{i, j \in \mathcal{A}} \pi(i) P_{i j} \log P_{i j}
$$


Uma prova deste teorema encontra-se em Cover e Thomas (1991).

Estudaremos a velocidade de convergência de $\frac{1}{k} H\left(\nu_{k}\right)$ e respectivamente de $h\left(\nu_{k+1}\right)$ para a entropia do processo na Seção 4.6.1.

Definição 3.1.12. Seja $\nu$ e $\mu$ duas medidas de probabilidades definidas sobre o mesmo espaço $\mathcal{A}^{\mathbb{Z}}$. Seja $k \geq 1$. Chamaremos $D\left(\nu_{k} \| \mu_{k}\right)$ de entropia relativa ou de distância de Kullback-Leibler, da medida $\nu_{k}$ com respeito à medida $\mu_{k}$ onde

$$
D\left(\nu_{k} \| \mu_{k}\right):=\sum_{a_{1}^{k} \in \mathcal{A}^{k}} \nu_{k}\left(a_{1}^{k}\right) \log \left(\frac{\nu_{k}\left(a_{1}^{k}\right)}{\mu_{k}\left(a_{1}^{k}\right)}\right) .
$$

Um estudo detalhado da entropia relativa é apresentado em Cover e Thomas (1991).

Na Seção 4.5 apresentaremos um resultado sobre a velocidade de convergência da entropia relativa para zero.

\subsection{Estimadores da entropia}

Para cada $n \geq 1$ consideraremos $x_{1}^{n} \in A^{n}$ uma amostra finita de um processo $\left\{X_{n}\right\}_{n \in \mathbb{Z}}$ e $k \leq n$.

Definição 3.2.1. Chamaremos de k-ésima distribuição empírica do processo, uma medida de probabilidade $\hat{\nu}_{k}$ sobre o espaço $A^{k}$ definida como

$$
\hat{\nu}_{k}\left(a_{1}^{k} \mid x_{1}^{n}\right):=\frac{\sum_{i=1}^{n-k+1} \mathbf{1}\left(x_{i}^{i+k-1}=a_{1}^{k}\right)}{n-k+1}, a_{1}^{k} \in A^{k}
$$

Por conveniência denotaremos às vezes $\hat{\nu}_{k}\left(\cdot \mid x_{1}^{n}\right)=\hat{\nu}_{k, n}$.

A partir da $k$-ésima distribuição empírica definida em (3.2.1) e das Definições 3.1.7 e 3.1.7, definiremos dois estimadores da entropia, isto é.

Definição 3.2.2. 1) Chamaremos $H\left(\hat{\nu}_{k}\left(\cdot \mid x_{1}^{n}\right)\right)$ de k-ésima entropia empírica.

2) Chamaremos $h\left(\hat{\nu}_{k+1}\left(\cdot \mid x_{1}^{n}\right)\right)$ de $k$-ésima entropia empírica condicionada.

Por conveniência denotaremos $\hat{H}_{k, n}=H\left(\hat{\nu}_{k}\left(\cdot \mid x_{1}^{n}\right)\right)$ e $\hat{h}_{k, n}=h\left(\hat{\nu}_{k+1}\left(\cdot \mid x_{1}^{n}\right)\right)$.

Observação 3.2.3. Consideraremos duas escalas diferentes, isto é, n que representa o comprimento da amostra e $k$ que representa o comprimento dos cilíndros considerados da medida $\nu$. O comportamento assintótico dos dois estimadores da entropia será analisado, na próxima Seção, separadamente segundo a relação existente entre as duas escalas. Consideramos em primeiro o caso quando $k$ é fixo em seguida, o caso em que $k$ é uma função crescente de $n$. 


\subsection{Resultados prévios}

\section{Caso $k$ fixo}

No caso onde $k$ não depende de $n$ a consistência dos estimadores da entropia, $\hat{H}_{k, n}$ e $\hat{h}_{k, n}$, é consequência do teorema ergódico clássico.. Isto é,

Proposição 3.3.1. Seja $x_{1}^{n} \in A^{n}$ uma amostra de um processo ergódico $\nu$. Para cada $k \geq 1$ valem $\nu$ quase-certamente as seguintes propriedades:

(i) para cada $a_{1}^{k} \in \mathcal{A}^{k}$

$$
\lim _{n \rightarrow \infty} \hat{\nu}_{k}\left(a_{1}^{k} \mid x_{1}^{n}\right)=\nu_{k}\left(a_{1}^{k}\right)
$$

(ii)

$$
\begin{aligned}
& \lim _{k \rightarrow \infty} \lim _{n \rightarrow \infty} \frac{1}{k} H\left(\hat{\nu}_{k}\left(\cdot \mid x_{1}^{n}\right)\right)=h(\nu) \\
& \lim _{k \rightarrow \infty} \lim _{n \rightarrow \infty} h\left(\hat{\nu}_{k+1}\left(\cdot \mid x_{1}^{n}\right)\right)=h(\nu) .
\end{aligned}
$$

Para cada $k$ fixo, Iosifescu (1965), provou que o estimador $\frac{1}{k} \hat{H}_{k, n}$ tem flutuações gaussianas em torno de $\frac{1}{k} H_{k}$, a $k$-ésima entropia do processo, e respectivamente o estimador $\hat{h}_{k, n}$ tem flutuações gaussianas em torno de $h_{k}$ a $k$-ésima entropia condicionada do processo.

Apresentaremos o resultado do Iosifescu somente para o estimador $\frac{1}{k} \hat{H}_{k, n}$. Lembramos que $\sigma_{f}$ foi definido em (2.3.2) e as funções $e_{k}$ foram definidas em (3.1.3).

\section{Teorema 3.3.2. (Iosifescu 1965)}

Seja $\left\{X_{n}\right\}_{n \in \mathbb{Z}}$ uma c.o.i.p.l.c., definida em (2.1.4), com taxa de perda de memória somável, isto é, $\sum_{i=1}^{\infty} \gamma_{i}<\infty$. Para cada $k$ fixado, $1 \leq k \leq n$ valem

- Se $\sigma_{e_{k}}^{2}>0$

$$
\lim _{n \rightarrow \infty} \mathbf{P}\left(\sqrt{\frac{n}{\sigma_{e_{k}}^{2}}}\left(\frac{1}{k} \hat{H}_{k}-\frac{1}{k} H_{k}\right)<\lambda\right)=\frac{1}{\sqrt{2 \pi}} \int_{-\infty}^{\lambda} e^{-u^{2} / 2} d u .
$$

uniformemente com respeito a $\lambda$;

- Se $\sigma_{e_{k}}^{2}=0$ então

$$
\sqrt{n}\left(\frac{1}{k} \hat{H}_{k, n}-\frac{1}{k} H_{k}\right) \stackrel{\mathrm{P}}{\rightarrow} 0 .
$$


Observação 3.3.3. Observamos em particular que se $\left\{X_{n}\right\}_{n \in \mathbb{Z}}$ for uma cadeia de Markov estacionária os estimadores considerados são estimadores da entropia do processo e flutuam, como variáveis aleatórias gaussianas, em torno da entropia do processo. A pergunta natural que temos agora é saber em geral, para c.o.i. como futuam os dois estimadores da entropia em torno da entropia do processo $e$ por outro lado como tem que ser a dependência entre as duas escalas $k$ e $n$. Este problema será considerado no próximo capítulo..

\section{Caso $\mathrm{k}=\mathrm{k}(\mathbf{n})$}

Ornstein e Weis (1990), provaram um Teorema ergódico que pode ser visto como um Teorema de Shanon-McMillan-Breiman para a medida empírica $\hat{\nu}_{k, n}$ quando $n$ cresce, para qualquer $k \leq \frac{1}{h} \log n$.

Teorema 3.3.4. (Ornstein-Weiss 1990) Seja um processo estacionário ergódico de medida $\nu$, e tal que $h(\nu)>0$. Para cada $k \geq 1$ existe um conjunto $T_{k}(\varepsilon) \in \mathcal{A}^{k}$ $\operatorname{com}\left|T_{k}(\varepsilon)\right| \leq 2^{n(h+\varepsilon)}$ e tal que para quase todos os $x \in \mathcal{A}^{\mathbb{Z}}$ existe $K=K(\varepsilon, n)$ tal que se $k \geq K$ e $n \geq 2^{k h}$ então

(i) $\hat{\nu}_{k, n}\left(T_{k}(\varepsilon)\right)>1-\varepsilon$;

(ii) $\hat{\nu}_{k, n}(B)<\varepsilon$, para qualquer $B \in \mathcal{A}^{k}$ satisfazendo $|B| \leq 2^{n(h-\varepsilon)}$.

Observaremos que para cada $k$ fixo o Teorema de Ornstein e Weiss é consequência do Teorema de Shanon-McMillan-Breiman e do Teorema ergódico clássico.

O Teorema de Ornstein e Weiss mostra que para quase todas as realizações do processo, dado $\varepsilon>0$ para todo $k$ suficientemente grande e $k \leq \frac{1}{h} \log n$ existe um conjunto $T_{k}(\varepsilon)$ de $k$-cilíndros tal que uma grande fração (com respeito a distribuição empírica), próxima de 1 , de $k$ - cilíndros da amostra pertencem a $T_{k}(\varepsilon)$ e por outro lado o número de $k$-cilíndros que pertencem a $T_{k}(\varepsilon)$ e de ordem $2^{k h}$.

Como consequência do Teorema precedente temos os seguintes resultados sobre a consistência dos dois estimadores da entropia considerados.

Teorema 3.3.5. (Ornstein e Weiss 1990)

Seja $\nu$ uma medida ergódica de entropia $h(\nu)>0$. Se $k(n) \leq \frac{1}{h(\nu)} \log n$, então $\nu$ quase-certamente vale

$$
\lim _{n \rightarrow \infty} \frac{1}{k(n)} H\left(\hat{\nu}_{k(n)}\left(\cdot \mid x_{1}^{n}\right)\right)=h(\nu)
$$

Este Teorema foi provado em Ornstein e Weiss(1990) e Shields(1996). 
Teorema 3.3.6. (Shields 2001)

Seja $\nu$ uma medida ergódica de entropia $h(\nu)>0$. Se $k(n)<\frac{1}{h(\nu)} \log n$, então v quase-certamente vale

$$
\lim _{n \rightarrow \infty} h\left(\hat{\nu}_{k(n)+1}\left(\cdot \mid x_{1}^{n}\right)\right)=h(\nu)
$$

Observação 3.3.7. Observamos, como consequência do Lema 4.3.1 ítem i) a seguir, que a entropia de uma c.o.i. positiva e log-contínua, que será considerada no próximo capítulo, é estritamente positiva. Então os resultados de Ornstein e Weiss (1990) e Shields (1990) valem em particular para c.o.i.p.l.c.. 


\section{Capítulo 4}

\section{Resultados novos. Flutuações da entropia empírica em torno da entropia do processo.}

Este capítulo contém os resultados originais desta tese que são os Teoremas $4.6 .4 \mathrm{e}$ 4.6.5. Eles são apresentados na Seção 4.6.2. Antes demonstraremos dois teoremas preliminares que são interessantes por si só. Usando a construção regenerativa, proposta em Comets, Fernández e Ferrari (2000), para cadeias de ordem infinita, provaremos um teorema-limite central (Teorema 4.1.1) para funções cilíndricas, ferramenta que usaremos para estudar as flutuações dos estimadores da entropia do processo. Usando o controle da velocidade de decaimentos das correlações, resultados apresentados em Bressaud, Fernández e Galves (1999 b), provaremos, no Teorema 4.5.1, um novo resultado sobre a velocidade de convergência para zero da distância de Kullback-Leibler, ver a equação (3.1.6), entre a $k(n)$-ésima distribuição empírica e a $k(n)$-ésima distribuição verdadeira, quando $n$, o comprimento da amostra, diverge.

Consideraremos o processo $\left(X_{n}, W_{n}\right)_{n \in \mathbb{Z}}$, definido na Seção 2.2 sobre o espaço de probabilidade $(\Omega, \Sigma, \mathbf{P})$. Denotaremos por $\mathbf{E}$ a esperança com respeito a $\mathbf{P}$. Considerarmos $\left(X_{n}\right)_{n \in \mathbb{Z}}$ uma cadeia de ordem infinita positiva, log-contínua com taxa de perda de memória exponencial, apresentados na Definição 2.1.4, com valores num alfabeto finito $\mathcal{A}$ e com probabilidade de transição $P$. Consideraremos $\nu$ a medida invariante do processo $\left(X_{n}\right)_{n \in \mathbb{Z}}$.

Para cada $n \geq 1$ consideraremos o vetor aleatório $X_{1}^{n}$ de comprimento $n$. e para $k \leq n$ consideraremos os $k$-cilíndros $X_{j-k}^{j-1}$ onde $k+1 \leq j \leq n$. $\mathrm{O}$ 
comprimento $k$ destes cilíndros $X_{j-k}^{j}$ será considerado crescente em $n$, isto é $k=$ $k(n)$. Consideraremos $\left(f_{k(n)}\right)_{n \geq 1}$ uma sequência de funções cilíndricas, $f_{k(n)}$ : $\mathcal{A}^{k(n)} \rightarrow \mathbb{R}$, satisfazendo as seguintes condições

i) $f_{k(n)} \in L^{2}(\nu)$;

ii) $\sup _{n \geq 1} \sup _{k(n)}\left\|f_{k(n)}\right\|_{+\infty}<+\infty$;

iii) para qualquer sequência $(s(n))_{n \geq 1}$ de inteiros positivos tal que $\lim _{n \rightarrow \infty} \frac{k(n)}{s(n)}=$ 0 a sequência $\left(\left(\sigma_{f_{k(n)}}^{2}\right)^{(s(n))}\right)_{n \geq 1}$ é convergênte. A sequência $\left(\left(\sigma_{f_{k(n)}^{2}}^{2}\right)^{m}\right)_{m \geq 1}$ foi definida na (2.3.3).

Definiremos

$$
\sigma^{2}:=\lim _{n \rightarrow \infty}\left(\sigma_{f_{k(n)}}^{2}\right)^{(s(n))}
$$

\subsection{Teorema-limite central}

Teorema 4.1.1. Seja $(k(n))_{n>1}$ uma sequência não decrescente de números, $1 \leq$ $k(n) \leq n$ tal que $\lim _{n \rightarrow \infty} k(n)=+\infty$. Para qualquer sequência de funções cilíndricas $\left(f_{k(n)}\right)_{n \geq 1}$ satisfazendo as condições precedentes valem

- Se $\sigma^{2}>0$ então

$$
\frac{\sum_{i=1}^{n}\left(T_{i} f_{k(n)}\left(X_{-k(n)}^{0}\right)-\mathbf{E}_{\nu}\left[f_{k(n)}\left(X_{-k(n)}^{0}\right)\right]\right)}{\sqrt{n \sigma^{2}}} \stackrel{\mathcal{D}}{\rightarrow} \mathcal{N}(0,1) .
$$

- Se $\sigma^{2}=0$ então

$$
\frac{\sum_{i=1}^{n}\left(T_{i} f_{k(n)}\left(X_{-k(n)}^{0}\right)-\mathbf{E}_{\nu}\left[f_{k(n)}\left(X_{-k(n)}^{0}\right)\right]\right)}{\sqrt{n}} \stackrel{\mathrm{P}}{\rightarrow} 0 .
$$

\section{Esboço da prova :}

Denotaremos

$$
s_{k(n), n}\left(X_{1}^{n}\right):=\sum_{i=1}^{n}\left(T_{i} f_{k(n)}\left(X_{-k(n)}^{0}\right)-\mathbf{E}_{\nu}\left[f_{k(n)}\left(X_{-k(n)}^{0}\right)\right]\right) .
$$

Por abuso de notação denotaremos $f_{k}:=f_{k}\left(X_{-k}^{0}\right)$ e $s_{k(n), n}:=s_{k(n), n}\left(X_{1}^{n}\right)$.

Segue da construção regenerativa de c.o.i.p.l.c. com perda de memória exponencial que existe uma sequência de tempos de regeneração do processo $\left(\tau_{i}\right)_{i \in \mathbb{Z}}$ tal que os vetores aleatórios $X_{\tau_{i}}^{\tau_{i+1}-1}$ são i.i.d. para todo $i \geq 1$, ver o Corolário 2.2.11. 
Queremos usar esta indepêndencia para demonstrar o teorema-limite central. Ingenuamente a idéia seria considerar apenas os $k(n)$-cilíndros que não contêm os tempos de regeneração. Entretanto, isto não é possível pois quando $n$ cresce o comprimento dos $k(n)$-cilíndros também divergem, enquanto a distância entre dois instantes de renovação tem distribuição majorada por uma geométrica com esperança finita. Ou seja, tipicamente qualquer cilíndro conterá mais de um tempo de renovação. Isto inviabiliza a estratégia ingênua. Uma solução para este problema será dizimar a sequência de instantes de renovação considerando para cada $n$ fixo os índices múltiplos de $s(n)$ onde a sequência $s(n)$ é escolhida estritamente crescente em $n$ e tal que a distância típica entre dois instantes de renovação sobreviventes seja muito maior que $k(n)$. Consideraremos por exemplo, $s(n)=k^{4}(n)$. Esta sequência verifica todas as condições requisitadas para a demonstração do teorema-limite central.

Então para cada $n \in \mathbb{Z}$ escolheremos uma nova sequência

$$
\tilde{\tau}_{i, n}:=\tau_{s(n) i}
$$

e denotaremos os incrementos

$$
\Delta_{\bar{\tau}_{i, n}}:=\tilde{\tau}_{i, n}-\tilde{\tau}_{i-1, n} .
$$

As propriedades do processo $\tilde{\tau}_{i, n}$ serão estudadas na Seção 4.2. Como consequência do Lema 4.2 .5 para cada $n \geq 1,\left(\tilde{\tau}_{n, i}\right)_{i \geq 0}$ é um novo processo de renovação e temos que $X_{\tilde{\tau}_{i, n}}^{\tilde{\tau}_{i+1, n}-1}$ são i.i.d para todo $i \geq 1$.

Para cada $n \geq 1$, definiremos as seguintes variáveis aleatórias

$$
\begin{aligned}
S_{(i, k(n), n)}^{\prime}: & =\sum_{j=\tilde{\tau}_{i, n}}^{\tilde{\tau}_{i+1, n}-k(n)-1}\left[T_{(j+k(n))} f_{k(n)}-\mathbf{E}_{\nu}\left[f_{k(n)}\right]\right] . \\
R_{(i, k(n), n)}^{\prime}: & =\sum_{j=\tau_{i, n}-k}^{\tilde{\tau}_{i, n}-1}\left[T_{(j+k(n))} f_{k(n)}-\mathbf{E}_{\nu}\left[f_{k(n)}\right]\right] .
\end{aligned}
$$

Por conveniência denotaremos por $S_{i}^{\prime}=S_{(i, k(n), n)}$ e $R_{i}^{\prime}=R_{(i, k(n), n)}^{\prime}$.

Observamos que para cada $i \geq 0$ os $k(n)$-cilindros que entram na expressão da variável aleatória $S_{i}^{\prime}$, não contêm o tempo de renovação $\tilde{\tau}_{i, n}$ e que os $k(n)$ cilindros que entram na expressão da variável aleatória $R_{(i, k(n), n)}^{\prime}$, contêm o tempo de renovação $\tilde{\tau}_{i, n}$. Consideraremos também as seguintes variáveis aleatórias

$$
\tilde{N}(n):=\sup _{i \geq 1}\left\{\tau_{s(n) i} \leq n\right\}=\sum_{j=1}^{+\infty} 1\left(\tilde{\tau}_{j, n} \leq n\right) .
$$




$$
\begin{aligned}
Q_{(\bar{N}(n), k(n))}:= & \sum_{j=1}^{\bar{\tau}_{0, n}+k(n)-1}\left[T_{j} f_{k(n)}-\mathbf{E}_{\nu}\left(f_{k(n)}\right)\right] \\
& -\sum_{j=n+1}^{\bar{\tau}_{\bar{N}(n)+1, n}+k(n)-1}\left[T_{j} f_{k(n)}-\mathbf{E}_{\nu}\left(f_{k(n)}\right)\right] .
\end{aligned}
$$

Usando as variáveis aleatórias definidas antes, escreveremos (4.1.1) como

$$
s_{k(n), n}=\sum_{i=0}^{\bar{N}(n)} S_{(i, k(n), n)}^{\prime}+\sum_{i=1}^{\bar{N}(n)+1} R_{(i, k(n), n)}^{\prime}+Q_{(\bar{N}(n), k(n))} .
$$

Consideraremos as seguintes variáveis aleatórias centralizadas

$$
S_{(i, k(n), n)}:=S_{(i, k(n), n)}^{\prime}-\mathrm{E} S_{(i, k(n), n)}^{\prime} .
$$

e

$$
R_{(i, k(n), n)}:=R_{(i, k(n), n)}^{\prime}-\mathrm{E} R_{(i, k(n), n)}^{\prime} .
$$

Por conveniência denotaremos por $S_{i}=S_{(i, k(n), n)}$ e $R_{i}=R_{(i, k(n), n)}$. Escreveremos (4.1.8) como

$$
\begin{aligned}
s_{k(n), n} & =\sum_{i=0}^{\tilde{N}(n)} S_{i}+\sum_{i=1}^{\tilde{N}(n)+1} R_{i} \\
& +Q_{(\tilde{N}(n), k(n))}+L_{(\tilde{N}(n), k(n))} .
\end{aligned}
$$

onde

$$
L_{(\tilde{N}(n), k(n))}:=(\tilde{N}(n)+1)\left(\mathrm{E} S_{i}^{\prime}+\mathrm{E} R_{i}^{\prime}\right) .
$$

Provaremos, ver o Lema 4.4.9, que a variável aleatória $\sum_{i=0}^{\bar{N}(n)} S_{i}$ converge em distribuição para uma variável gaussiana e que todos os outros termos do membro direito da igualdade (4.1.11) convergem em probabilidade para zero. Na Seção 4.4 apresentaremos uma prova do Teorema 4.1.1. As Seções 4.2 e 4.3 contêm resultados auxiliares que usaremos na Seção 4.4. 


\subsection{Regeneração. Propriedades}

Consideraremos $\left(X_{n}\right)_{n \in \mathbb{Z}}$ uma c.o.i.p.l.c. com perda de memória exponencial construída segundo esquema regenerativo apresentado em Comets, Fernández e Ferrari (2000).(Ver o Corolário 2.2.11.) Consideraremos $\left(\tau_{i}\right)_{i \geq-1}$ o processo de renovação estacionário, definido em (2.2.3), $\operatorname{com} \gamma:=\mathrm{E}\left(\triangle_{\tau_{0}}\right)$. Consideraremos uma familia de cadeias castelo de cartas $\left\{\left(Z_{n}^{0, t}\right)_{n \geq t}\right\}_{t \in \mathbb{Z}}$ com as probabilidades de transição definidas em (2.2.4) e commeçãndo na origem no tempo $t \in \mathbb{Z}$. Acoplaremos essas cadeias usando a sequência de variáveis aleatórias uniformes $U$, isto é,

$$
Z_{n}^{0, t}= \begin{cases}0, & n \leq t \\ \left(Z_{n-1}^{0, t}+1\right) 1\left\{U_{n} \leq \alpha_{Z_{n-1}^{0, t}}\right\}, & n \geq t+1\end{cases}
$$

Observamos que o processo definido em (2.2.4) é tal que $\left(Z_{n}\right)_{n \in \mathbb{Z}}=\left(Z_{n}^{0,0}\right)_{n \in \mathbb{Z}}$.

Definição 4.2.1. Sejam $\left(X_{n}\right)_{n \in \mathbb{Z}}$ uma c.o.i. de probabilidade de transição $P e$ $\left(\alpha_{k}\right)_{k \geq 0}$ a sequência da Definição 2.2.1. Chamaremos, a familia de processos (4.2.1), de cadeia de castelo de cartas associada ao processo $\left(X_{n}\right)_{n \in \mathbb{Z}}$.

Lema 4.2.2. Sejam $\left\{\left(Z_{n}^{0, t}\right)_{n \in \mathbb{Z}}\right\}_{t \in \mathbb{Z}}$ o castelo de cartas associado ao processo $\left(X_{n}\right)_{n \in \mathbb{Z}}$ e $\tau[l, m]$ o tempo de regeneração de uma janela $[l, m]$, definido em (2.2.1). Vale a seguinte identidade:

$$
\{\tau[l, m]<t\}=\cup_{i \in[l, m]}\left\{Z_{i}^{0, t}=0\right\} .
$$

parat $\leq l$.

Uma prova deste resultado se encontra no livro de Fernández, Ferrari, Galves (2001).

Observação 4.2.3. Segue de (2.2.1) que

$$
\{\tau[t,+\infty]=t\}=\cap_{j \geq t}\left\{Z_{j}^{0, t-1}>0\right\} .
$$

Lema 4.2.4. Sejam $f: \mathcal{A}^{Z} \rightarrow \mathbb{R}$ uma função cilíndrica. Para todo $j \in \mathbb{Z}$ e para todo $l \leq j$ as variáveis aleatórias $\Delta_{\tau_{l}}$ e $f\left(X_{\tau_{j}}^{+\infty}\right)$ são independentes.

Prova. Temos

$$
\mathrm{P}\left(\Delta_{\tau_{l}} \geq m, f\left(X_{\tau_{j}}^{+\infty}\right)=r\right)=\sum_{t} \mathrm{P}\left(\Delta_{\tau_{l}} \geq m, f\left(X_{\tau_{j}}^{+\infty}\right)=r, \tau_{l}=t\right) .
$$




\section{Escreveremos}

$$
\left\{\Delta_{\tau_{l}} \geq m, f\left(X_{\tau_{j}}^{+\infty}\right)=r, \tau_{l}=t\right\}=A_{t} \cap\left\{f\left(X_{\tau_{j}}^{+\infty}\right)=r, \tau_{l}=t\right\}
$$

onde

$$
\begin{aligned}
A_{t} & :=\left\{\Delta_{\tau_{l}} \geq m, \tau_{l}=t\right\} \\
& =\left\{\tau[t-1,+\infty]<t-m+1, \tau[t,+\infty]=t, \tau_{l}=t\right\} \\
& =\left\{\cup_{j \geq t-1} Z_{j}^{0, t-m}=0\right\} \cap\left\{\cap_{j \geq t} Z_{j}^{0, t-1}>0\right\} \cap\left\{\tau_{l}=t\right\}
\end{aligned}
$$

Como $Z_{j}^{0, t-1} \leq Z_{j}^{0, t-m}$ para todo $j \geq t$ e como $\left\{\tau_{l}=t\right\} \subseteq\{\tau[t,+\infty]=t\}$ temos então

$$
\begin{aligned}
A_{t} & =\left\{Z_{t-1}^{0, t-m}=0\right\} \cap\left\{\cap_{j \geq t} Z_{j}^{0, t-1}>0\right\} \cap\left\{\tau_{l}=t\right\} \\
& =\left\{Z_{t-1}^{0, t-m}=0\right\} \cap\left\{\tau_{l}=t\right\} .
\end{aligned}
$$

Em conclusão temos

$$
\left\{\Delta_{\tau_{l}} \geq m, f\left(X_{\tau_{j}}^{+\infty}\right)=r, \tau_{l}=t\right\}=\left\{Z_{t-1}^{0, t-m}=0\right\} \cap\left\{f\left(X_{\tau_{j}}^{+\infty}\right)=r, \tau_{l}=t\right\} .
$$

Observaremos que

$$
\left\{Z_{t-1}^{0, t-m}=0\right\} \in \Sigma\left(U_{t-m}^{t-1}\right)
$$

Por outro lado para todo $l \leq j$ temos $\tau_{l} \leq \tau_{j}$, então

$$
\left\{f\left(X_{\tau_{j}}^{+\infty}\right)=r, \tau_{l}=t\right\} \in \Sigma\left(U_{t}^{+\infty}\right)
$$

Seque de (4.2.4), (4.2.5) e (4.2.6) que para todo $t \in \mathbb{Z}$ vale

$$
\begin{aligned}
\mathbf{P}\left(\Delta_{\tau_{l}} \geq m \mid \tau_{l}=t\right) & =\mathbf{P}\left(A_{t} \mid \tau[t,+\infty]=t\right) \\
& =\mathbf{P}\left(Z_{t-1}^{0, t-m}=0\right)=\gamma_{m}^{*} .
\end{aligned}
$$

Como consequência temos

$$
\begin{aligned}
\mathbf{P}\left(\Delta_{\tau_{l}} \geq m\right) & =\sum_{t} \mathbf{P}\left(\Delta_{\tau_{l}} \geq m \mid \tau_{l}=t\right) \mathbf{P}\left(\tau_{l}=t\right) \\
& =\mathbf{P}\left(Z_{t-1}^{0, t-m}=0\right)=\gamma_{m}^{*}
\end{aligned}
$$


Então segue de (4.2.3), (4.2.4), (4.2.5), (4.2.6)

$$
\begin{aligned}
\mathrm{P}\left(\triangle_{\tau_{l}} \geq m, f\left(X_{\tau_{j}}^{+\infty}\right)=r\right) & =\sum_{t} \mathbf{P}\left(Z_{t-1}^{0, t-m}=0\right) \mathbf{P}\left(f\left(X_{\tau_{j}}^{+\infty}\right)=r, \tau_{l}=t\right) \\
& =\mathbf{P}\left(Z_{t-1}^{0, t-m}=0\right) \sum_{t} \mathbf{P}\left(f\left(X_{\tau_{j}}^{+\infty}\right)=r, \tau_{l}=t\right) \\
& =\mathbf{P}\left(\triangle_{\tau_{l}} \geq m\right) \mathbf{P}\left(f\left(X_{\tau_{j}}^{+\infty}\right)=r\right) .
\end{aligned}
$$

Isto conclui a prova do Lema.

Para cada $n \geq 1$ consideraremos a variável aleatória

$$
N(n):=\sup _{i \geq 0}\left\{\tau_{i} \leq n\right\}
$$

Consideraremos o processo $\left(\tilde{\tau}_{n, i}\right)_{i \in Z}$ definido em (4.1.2) e as variáveis aleatórias $\Delta_{\tilde{\tau}_{n, i}}$ definidas em (4.1.3).

Lema 4.2.5. 1) Para cada $n \geq 1$, $\left(\tilde{\tau}_{i, n}\right)_{i \geq 0}$ é um processo de renovação estacionário e para todo $m \geq 1$ valem as seguintes propriedades

$$
\begin{array}{r}
\mathbf{E}\left(\Delta_{\tilde{\tau}_{i, n}}\right)=O(s(n)) ; \\
\mathbf{E}\left(\Delta_{\tilde{\tau}_{i, n}}-\mathbf{E}\left(\Delta_{\tilde{\tau}_{i, n}}\right)\right)^{2}=O(s(n)) \\
\mathbf{E}\left(\Delta_{\tilde{\tau}_{i, n}}\right)^{m}=O\left(s^{m}(n)\right)
\end{array}
$$

2) Para cada $n \geq 1,\left(\tau_{i, n}^{*}\right)_{i \in \mathbb{Z}}$ é um processo de renovação estacionário, onde $\tau_{i, n}^{*}:=\tau_{s(n) i-k(n)}$ para cada $i \in Z$.

Prova. Observamos que

$$
\begin{aligned}
\Delta \tilde{\tau}_{i, n} & =\sum_{j=0}^{s(n)-1} \Delta \tau_{s(n) i-j} . \\
\Delta \tilde{\tau}_{i, n}^{*} & =\sum_{j=0}^{s(n)-1} \Delta \tau_{s(n) i-j-k(n)} .
\end{aligned}
$$

Como $\left(\tau_{i}\right)_{i \geq-1}$ é um processo de renovação estacionário podemos concluir a prova do lema. 
Lema 4.2.6. Para as sequências de variáveis aleatórias $(N(n))_{n \geq 1} e(\tilde{N}(n))_{n \geq 1}$, definidas em (4.2.9) e (4.1.6). Valem as seguintes propriedades

i)

$$
\begin{gathered}
\frac{1}{n} N(n) \stackrel{\mathrm{P}}{\rightarrow} \frac{1}{\gamma} . \\
\lim _{n \rightarrow \infty} \mathrm{E}\left(\frac{1}{n} N(n)\right)=\frac{1}{\gamma} .
\end{gathered}
$$

ii) $S e \lim _{n \rightarrow \infty} \frac{s(n)}{n}=0$ então

$$
\begin{gathered}
\frac{s(n)}{n} \tilde{N}(n) \stackrel{\mathbf{P}}{\rightarrow} \frac{1}{\gamma} . \\
\lim _{n \rightarrow \infty} \mathbf{E}\left(\frac{s(n)}{n} \tilde{N}(n)\right)=\frac{1}{\gamma} .
\end{gathered}
$$

Prova. i) Segue da teoria clássica sobre os processos de renovação (ver, por exemplo, Grimmett \& Stirzaker (1992)).

ii) Como consequências das definições (4.2.9) e (4.1.6) temos por um lado que $s(n) \tilde{N}(n) \leq N(n)$ e por outro lado temos $\left[\frac{N(n)}{s(n)}\right] \leq \tilde{N}(n)$. Então

$$
N(n)-s(n) \leq \tilde{N}(n) s(n) \leq N(n) .
$$

Usando (i) concluimas a prova do (ii).

Consideraremos $\mu_{C}$ a lei conjunta do processo $\left(X_{n}, W_{n}\right)_{n \in \mathbb{Z}}$ construido na Seção 2.2. Vamos estimar a diferença $D_{j}(f)$, definida em (4.4.7), entre $\mathbf{E}_{\mu_{G}}\left(T_{\tau_{0}+j} f\right)$ e $\mathbf{E}_{\mu_{C}}(f)$ e a diferença entre a variância da função $T_{\bar{\tau}_{0}^{n}} f$ e a varianciia da funç ão $f$.Lembramos (ver o Teorema 2.2 .10 e a equação(2.2.2)), que $\left(W_{n}\right)_{n \in \mathbb{Z}}$ é um processo estacionário de renovação com valores em $\{0,1\}^{\mathbb{Z}}$ e tal que $\mathbf{P}\left(W_{j}=1\right)=$ $\beta$.

Lema 4.2.7. Seja $f=f\left(a_{-k}^{0}\right): A^{k+1} \rightarrow \mathbb{R}$ uma função cilíndrica e limitada. Valem as seguintes propriedades:

1) Para todo $j \geq 1$

$$
\left|D_{j}(f)\right| \leq \sum_{t_{0}=1}^{+\infty} \beta(1-\beta)^{t_{0}} \operatorname{var}_{j-t_{0}}(f) .
$$


Onde $\operatorname{var}_{j}(f)$, foi definida em (2.3.6).

2) $\mathrm{Se} \sum_{j=0}^{+\infty} \operatorname{var}_{j}(f)<+\infty$ então para todo $m \geq 1$ e para todo $j \geq 0$

$$
\sum_{j=0}^{m}\left|D_{j}(f)\right|<+\infty \text {. }
$$

3) Se $\sum_{j=0}^{+\infty} \operatorname{jvar}_{j}(f)<+\infty$ então para todo $m \geq 1$ e para todo $j \geq 0$ existe uma constante $M$ tal que

$$
\sum_{d=1}^{m-1} \sum_{j=d+1}^{m}\left|D_{j}\left[(f-\mathbf{E} f) T_{-d}(f-\mathbf{E} f)\right]\right| \leq M m .
$$

Prova. Para todo $i \neq-1$, os vetores aleatórios $X_{\bar{\tau}_{i, n}}^{\bar{\tau}_{i+1, n}-1}$ são identicamente distribuídos então para todo $t, j \geq 1$ temos

$$
\mathbf{E}\left(T_{\bar{\tau}_{i, n}+j} f\right)=\mathbf{E}_{\mu_{C}\left(\cdot \mid W_{0}=1\right)}\left(T_{j} f\right) .
$$

Para provar essa afirmação observaremos que para todo cilíndro $a_{-k}^{0}$ e para todo $t \in \mathbb{Z}$ temos

$$
\mathbf{P}\left(X_{\tilde{\tau}_{i, n}+j-k}^{\tilde{\tau}_{i, n}+j}=a_{-k}^{0} \mid \tilde{\tau}_{i, n}=t\right)=\mathbf{P}\left(X_{t+j-k}^{t+j}=a_{-k}^{0} \mid W_{t}=1\right) .
$$

Usando a estacionáridade do processo $\left(X_{t}, W_{t}\right)_{t \in \mathbb{Z}}$ temos

$$
\mathbf{P}\left(X_{\tilde{\tau}_{i, n}+j-k}^{\tilde{\tau}_{i, n}+j}=a_{-k}^{0} \mid \tilde{\tau}_{i, n}=t\right)=\mathbf{P}\left(X_{j-k}^{j}=a_{-k}^{0} \mid W_{0}=1\right) .
$$

Então para todo $t \in \mathbb{Z}$ vale

$$
\mathbf{E}\left(T_{\tilde{\tau}_{i, n}+j} f \mid \tilde{\tau}_{i, n}=t\right)=\mathbf{E}\left(T_{j} f \mid W_{0}=1\right) .
$$

Em consequência, temos

$$
\begin{aligned}
\mathbf{E}\left(T_{\tilde{\tau}_{i, n}+j} f\right) & =\sum_{t \in \mathbb{Z}} \mathbf{E}\left(T_{\tilde{\tau}_{i, n}+j} f \mid \tilde{\tau}_{i, n}=t\right) \mathbf{P}\left(\tilde{\tau}_{i, n}=t\right) \\
& =\mathbf{E}\left(T_{j} f \mid W_{0}=1\right) .
\end{aligned}
$$

Então

$$
\begin{aligned}
D_{j}(f) & =\mathbf{E}_{\mu_{C}\left(\cdot \mid W_{0}=1\right)}\left(T_{j} f\right)-\mathbf{E}_{\mu_{C}}(f) \\
& =\mathbf{E}_{\mu_{C}\left(\cdot W_{0}=1\right)}\left(T_{j} f\right)-\beta \mathbf{E}_{\mu_{C}\left(\cdot \mid W_{0}=1\right)}\left(T_{j} f\right)-(1-\beta) \mathbf{E}_{\mu_{C}\left(\cdot \mid W_{0}=0\right)}\left(T_{j} f\right) \\
& =(1-\beta)\left(\mathbf{E}_{\mu_{C}\left(\cdot \mid W_{0}=1\right)}\left(T_{j} f\right)-\mathbf{E}_{\mu_{C}\left(\cdot W_{0}=0\right)}\left(T_{j} f\right)\right) .
\end{aligned}
$$


Observamos que para qualquer $l \in\{0,1\}$ temos

$$
\begin{aligned}
\mathbf{E}_{\mu_{C}\left(\cdot \mid W_{0}=l\right)}\left(T_{j} f\right) & =\sum_{t_{0} \geq 1} \mathbf{E}_{\mu_{C}\left(\cdot \mid W_{0}=l, \tau_{0}=t_{0}\right)}\left(T_{j} f\right) \mathbf{P}\left(\tau_{0}=t_{0} \mid W_{0}=l\right) \\
& =\sum_{t_{0} \geq 1} \beta(1-\beta)^{t_{0}-1} \mathbf{E}_{\mu_{C}\left(\cdot \mid W_{0}=l, \tau_{0}=t_{0}\right)}\left(T_{j} f\right) .
\end{aligned}
$$

Então

$$
D_{j}(f)=\beta \sum_{t_{0}=1}^{+\infty}(1-\beta)^{t_{0}} D_{j, t_{o}}(f)
$$

onde

$$
D_{j, t_{0}}(f):=\mathbf{E}_{\mu_{C}\left(\cdot \mid W_{0}=1, \tau_{0}=t_{0}\right)}\left(T_{j} f\right)-\mathbf{E}_{\mu_{C}\left(\cdot \mid W_{0}=0, \tau_{0}=t_{0}\right)}\left(T_{j} f\right) .
$$

Provaremos que para cada $t_{0} \geq 1$ vale

$$
\left|D_{j, t_{0}}(f)\right| \leq \operatorname{var}_{j-t_{0}}(f) .
$$

Suponhamos, inicialmente, que $t_{0} \leq j$.

Segue da construção regenerativa das cadeias de ordem infinita $\left\{X_{n}\right\}_{n \in \mathbb{Z}}$ ( ver 2.2.11), que os vetores aleatórios $\left(X_{\tilde{\tau}_{0, n}}, \ldots, X_{\tilde{\tau}_{1, n}-1}\right)$ são independentes das variáveis aleatórias $\left\{X_{i}: i<\tilde{\tau}_{0, n}\right\}$. Então

$$
\begin{aligned}
& \mu_{C}\left(\left[a_{-k+j}^{j}\right] \mid W_{0}=1, \tau_{0}=t_{0}\right)=\mu\left(\left[a_{t_{0}}^{j}\right]\right) \mu^{1}\left(\left[a_{-k+j}^{t_{0}-1}\right],\right. \\
& \mu_{C}\left(\left[a_{-k+j}^{j}\right] \mid W_{0}=0, \tau_{0}=t_{0}\right)=\mu\left(\left[a_{t_{0}}^{j}\right]\right) \mu^{2}\left(\left[a_{-k+j}^{t_{0}-1}\right]\right) .
\end{aligned}
$$

Onde $\mu^{1}, \mu^{2}, \mu$ são algumas medidas de probabilidade.

$$
D_{j, t_{o}}(f)=\sum_{a_{t_{0}}^{j}} \mu\left(\left[a_{t_{0}}^{j}\right]\right) A\left(a_{t_{0}}^{j}\right)
$$

onde

$$
A\left(a_{t_{0}}^{j}\right):=\sum_{\substack{a_{0}-1 \\ a_{-k+j}}} \mu^{1}\left(\left[a_{-k+j}^{t_{0}-1}\right]\right) f\left(a_{-k+j}^{j}\right)-\sum_{\substack{t_{0}-1 \\ a_{-k+j}}} \mu^{2}\left(\left[a_{-k+j}^{t_{0}-1}\right]\right) f\left(a_{-k+j}^{j}\right) .
$$


Observamos que

$$
\begin{aligned}
& \left|A\left(a_{t_{0}}^{j}\right)\right| \leq \sup _{\substack{t_{0}-1 \\
a_{-k+j}}} f\left(a_{-k+j}^{j}\right)-\inf _{a_{-k+j}^{t_{0}-1}} f\left(a_{-k+j}^{j}\right) \\
& \leq \sup _{\substack{\dot{a}_{t_{0}}^{j} \\
a_{-k+j}}}\left(\sup _{\substack{t_{0}-1 \\
a_{-k+j}}} f\left(a_{-k+j}^{j}\right)-\inf _{a_{-k+j}^{t_{0}-1}} f\left(a_{-k+j}^{j}\right)\right) \\
& =\operatorname{var}_{j-t_{0}}(f)
\end{aligned}
$$

Então, como consêquencia de (4.2.23) e (4.2.24) temos

$$
\left|D_{j, t_{o}}(f)\right| \leq \operatorname{var}_{j-t_{0}}(f)
$$

Se $t_{o} \geq j+1$ temos

$$
\begin{aligned}
\left|D_{j, t_{o}}(f)\right| & \leq \sum_{a_{j-k}^{j}} \mu_{C}\left(\left[a_{j-k}^{j}\right] \mid W_{0}=1, \tau_{0}=t_{0}\right) f\left(a_{-k+j}^{j}\right) \\
& -\sum_{a_{-k+j}^{j}} \mu_{C}\left(\left[a_{-k+j}^{j}\right] \mid W_{0}=0, \tau_{0}=t_{0}\right) f\left(a_{-k+j}^{j}\right) \\
& \leq \sup _{a_{-k+j}^{j}} f\left(a_{-k+j}^{j}\right)-\inf _{a_{-k+j}^{j}} f\left(a_{-k+j}^{j}\right)=\operatorname{var}_{-1}(f) . \\
& \leq\|f\|_{\infty} .
\end{aligned}
$$

Em consequência, via (4.2.19), (4.2.25) e (4.2.26) podemos concluir a prova do primeiro ítem do Lema.

2)Podemos estender o Lema 4.2 .7 para fonções não cilíndricas. Então usando o primeiro ítem do lema temos

$$
\begin{aligned}
\sum_{j=1}^{m}\left|D_{j}(f)\right| & \leq M \sum_{j=0}^{m} \sum_{t_{0}=1}^{+\infty} \beta(1-\beta)^{t_{0}} \operatorname{var}_{j-t_{0}}(f) \\
& \leq M \sum_{t_{0}=1}^{+\infty} \beta(1-\beta)^{t_{0}}\left(\sum_{j=1}^{t_{0}} \operatorname{var}_{j-t_{0}}(f)+\sum_{j=t_{0}+1}^{+\infty} \operatorname{var}_{j-t_{0}}(f)\right) \\
& \leq M\|f\|_{\infty} \sum_{t_{0}=1}^{+\infty} t_{0} \beta(1-\beta)^{t_{0}}+M \sum_{j=1}^{+\infty} \operatorname{var}_{j}(f) \sum_{t_{0}=1}^{+\infty} \beta(1-\beta)^{t_{0}} \\
& <+\infty .
\end{aligned}
$$


3) Para todo $m \geq d$, usando a Definição 2.3.6, vale

$$
\begin{aligned}
V & :=\operatorname{var}_{m}\left[(f-\mathrm{E} f) T_{-d}(f-\mathrm{E} f)\right] \\
& =\sup _{a \underline{m}}\left|(f(a)-\mathrm{E} f) T_{-d}(f(a)-\mathrm{E} f)-(f(b)-\mathrm{E} f) T_{-d}(f(b)-\mathrm{E} f)\right| \\
& \leq \sup _{a_{\underline{m}} b}\left|(f(a)-f(b))\left(T_{-d} f(a)-\mathrm{E} f\right)\right| \\
& +\sup _{a \underline{\underline{m}} b}\left|\left(T_{-d} f(a)-T_{-d} f(b)\right)(f(b)-\mathrm{E} f)\right| \\
& \leq 2\|f\|_{\infty}\left(\sup _{a \stackrel{m}{=} b}|(f(a)-f(b))|+\sup _{a \stackrel{m}{=} b}\left|\left(T_{-d} f(a)-T_{-d} f(b)\right)\right|\right) \\
& =2\|f\|_{\infty}\left(\operatorname{var}_{m}(f)+\operatorname{var}_{m-d}(f)\right) .
\end{aligned}
$$

Se $m \leq d-1$ vale

$$
\operatorname{var}_{m}\left[(f-\mathbf{E} f) T_{-d}(f-\mathbf{E} f)\right] \leq 8\|f\|_{\infty} .
$$

Notaremos $\tilde{f}:=(f-\mathbf{E} f) T_{-d}(f-\mathbf{E} f)$.

Usando (4.2.16)temos

$$
\begin{aligned}
\sum_{d=0}^{m-1} \sum_{j=d+1}^{m}\left|D_{j}(\tilde{f})\right| & \leq \sum_{d=0}^{m-1} \sum_{j=d+1}^{m} \sum_{t_{0} \geq 1}^{m} \beta(1-\beta)^{t_{0}} \operatorname{var}_{j-t_{0}}(\tilde{f}) \\
& \leq \sum_{t_{0} \geq 1} \sum_{d=1}^{m-1} \sum_{j=d+1}^{d+t_{0}-1} \beta(1-\beta)^{t_{0}} \operatorname{var}_{j-t_{0}}(\tilde{f}) \\
& +\sum_{t_{0} \geq 1} \sum_{d=0}^{m-1} \sum_{j=d+t_{0}+1}^{+\infty} \beta(1-\beta)^{t_{0}} \operatorname{var}_{j-t_{0}}(\tilde{f})
\end{aligned}
$$

Para cada um dos termos do membro direito da desigualdade precedente, provaremos, para cada $k$ e $n$, que e limitado por uma constante vezes $m$. Isto é, usando (4.2.28) temos

$$
\begin{aligned}
A: & =\sum_{t_{0} \geq 1} \sum_{d=0}^{m-1} \sum_{j=d+1}^{d+t_{0}-1} \beta(1-\beta)^{t_{0}} \operatorname{var}_{j-t_{0}}(\tilde{f}) \\
& \leq 8 m\|f\|_{\infty} \sum_{t_{0}=1}^{+\infty} \beta t_{0}(1-\beta)^{t_{0}} .
\end{aligned}
$$


Usando (4.2.27) temos

$$
\begin{aligned}
B: & =\sum_{t_{0} \geq 1} \sum_{d=0}^{m-1} \sum_{j=d+t_{0}+1}^{+\infty} \beta(1-\beta)^{t_{0}} \operatorname{var}_{j-t_{0}}(\tilde{f}) \\
& \leq 2\|f\|_{\infty} \sum_{t_{0} \geq 1} \sum_{d=0}^{m-1} \sum_{j=d+t_{0}+1}^{+\infty} \beta(1-\beta)^{t_{0}}\left(\operatorname{var}_{j-t_{0}}(f)+\operatorname{var}_{j-t_{0}-d}(f)\right) \\
& \leq M\left(\sum_{j=0}^{+\infty} j \operatorname{var}_{j}(f)\right) \sum_{t_{0}=1}^{+\infty} \beta(1-\beta)^{t_{0}} \\
& +m M\left(\sum_{j=0}^{+\infty} \operatorname{var}_{j}(f)\right) \sum_{t_{0}=1}^{+\infty} \beta(1-\beta)^{t_{0}}
\end{aligned}
$$

Então, sobre a hipótese do item 3 do lema temos $B \leq M m$. Isto conclui a prova do lema.

Corolario 4.2.8. Sejam $n, k \geq 1$ e $f=f\left(a_{-k}^{0}\right): A^{k+1} \rightarrow \mathbb{R}$ uma função cilíndrica e limitada. Para tudo $m \geq 1$ valem

1) $\operatorname{Se} \sum_{j=0}^{+\infty} \operatorname{jvar}_{j}(f)<+\infty$ então existe uma constante $M$ tal que

$$
\left|\mathbf{E}\left(\sum_{j=0}^{m}\left(T_{\tilde{\tau}_{i}^{n}+j} f-\mathbf{E} f\right)\right)^{2}-m\left(\sigma_{f}^{2}\right)^{m}\right| \leq M m .
$$

2)

$$
\left|\mathbf{E}\left(\sum_{j=0}^{m}\left(T_{\tilde{\tau}_{i}^{n}+j+k} f-\mathbf{E} f\right)\right)^{2}-m\left(\sigma_{f}^{2}\right)^{m}\right|<+\infty
$$

Prova. Usando a notação $\tilde{f}=f-\mathbf{E} f$ escreveremos para cada $l \geq 0$

$$
\begin{aligned}
\left(\sum_{j=0}^{m}\left(T_{\tilde{\tau}_{i}^{n}+j+l} f-\mathrm{E} f\right)\right)^{2} & =\sum_{j=0}^{m} \sum_{j^{\prime}=0}^{m}\left[T_{\tilde{\tau}_{i}^{n}+j+l} \tilde{f}\right]\left[T_{\tilde{\tau}_{i}^{n}+j^{\prime}+l} \tilde{f}\right] \\
& =\sum_{j=0}^{m} T_{\tilde{\tau}_{i}^{n}+j+l} \tilde{f}^{2}+2 \sum_{d=0}^{m-1} \sum_{j=d+1}^{m} T_{\tilde{\tau}_{i}^{n}+j+l}\left[\tilde{f} T_{-d} \tilde{f}\right] .
\end{aligned}
$$


Usando a notação de $\left(\sigma_{f}^{2}\right)^{m}$ introduzida em (2.3.3), escreveremos

$$
\begin{aligned}
L & :=\mathbf{E}\left(\sum_{j=0}^{m}\left(T_{\tilde{\tau}_{i}^{n}+j+l} f-\mathbf{E} f\right)\right)^{2}-m\left(\sigma_{f}^{2}\right)^{m} \\
& =\sum_{j=0}^{m}\left(\mathrm{E}\left[T_{\tilde{\tau}_{i}^{n}+j+l} \tilde{f}^{2}\right]-\mathbf{E}\left[\tilde{f}^{2}\right]\right) \\
& +2 \sum_{d=0}^{m-1} \sum_{j=d+1}^{m}\left(\mathrm{E}\left[T_{\tilde{\tau}_{i}^{n}+j+l}\left(\tilde{f} T_{-d} \tilde{f}\right)\right]-\mathbf{E}\left[\tilde{f} T_{-d} \tilde{f}\right]\right)
\end{aligned}
$$

Usando as notações do Lema 4.2.7 podemos escrever

$$
L=\sum_{j=0}^{m} D_{j+l}\left(\tilde{f}^{2}\right)+2 \sum_{d=0}^{m-1} \sum_{j=d+1}^{m} D_{j+l}\left(\tilde{f} T_{-d} \tilde{f}\right) .
$$

Observamos que para $l=0$ se $\sum_{j=0}^{+\infty} \operatorname{jvar}_{j}(f)<+\infty$ usando o lema precedente podemos concluir o primeiro ítem do lema.

Do outro lado segue do Lema 4.2.7 que

$$
\left|D_{j+k}(f)\right| \leq M(1-\beta)^{j+1} .
$$

Então temos

$$
\begin{array}{r}
\sum_{j=0}^{m}\left|D_{j+k}(f)\right|<+\infty . \\
\sum_{d=0}^{m-1} \sum_{j=d+1}^{m}\left|D_{j+k}(f)\right|<+\infty .
\end{array}
$$

Isto conclui a prova do Corolário.

\subsection{Covariâncias}

Consideraremos $\nu$ a medida invariante de um processo estacionário com valores em $\mathcal{A}$. Usaremos as notações e as definições das covariâncias introduzidas na Seção 2.3.2. Consideraremos as funções $\phi, \phi_{k}$ e $e_{k} \operatorname{com} k \geq 1$ definidas em (3.1.3) e $I_{\left[a_{1}^{k}\right]}$ a função indicadora de um $k$-cilindrondro $\left[a_{1}^{k}\right] \in A^{Z}, k \geq 1$. Consideraremos $v a r_{m} f$, a variação da função $f$, definida em (2.3.6). 


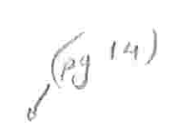

Lema 4.3.1. Se $\left(X_{n}\right)_{n \in \mathbb{Z}}$ for uma c.o.i.p.l.c. com taxa de perda de memória $\left(\gamma_{k}\right)_{k \in \mathbb{N}}$ então

i) As funções $\phi, \phi_{k}$, e e são estritamente positivas e uniformemente limitadas.

ii) Existem duas constantes $M$ e $M^{\prime}$ tal que $M^{\prime} \gamma_{k} \leq \operatorname{var}_{k}(\phi) \leq M \gamma_{k}$.

iii) Para todo $m \leq k$, $\operatorname{var}_{m}\left(\phi_{k}\right) \leq \gamma_{k}$ e para $m \geq k$, $\operatorname{var}_{m}\left(\phi_{k}\right)=0$.

iv) Para todo $k \geq 1$ vale

$$
\left\|\phi-\phi_{k}\right\|_{\infty}=O\left(\gamma_{k}\right)
$$

v) Para todo $i, j \geq 1$ existe $M^{\prime \prime}$ tal que

$$
\left\|\phi_{i}-\phi_{j}\right\|_{\infty} \leq M^{\prime \prime} \gamma_{\mathrm{inf}\{i, j\}}
$$

Prova. Seque da definição da cadeia considerada, que

$$
0<\inf _{x} \nu\left(x_{0} \mid x_{-\infty}^{-1}\right) \leq \nu\left(x_{0} \mid x_{-\infty}^{-1}\right) \leq \sup _{x} \nu\left(x_{0} \mid x_{-\infty}^{-1}\right)<1 .
$$

então $\phi$ é estritamente positiva e uniformemente limitada. Como consêquencia de (2.3.2) temos também, que $\phi_{k}$ é estritamente positiva e uniformemente limitada. Consideraremos as seguintes constantes

$$
c_{M}:=-\log \left(\inf _{x} \nu\left(x_{0} \mid x_{-\infty}^{-1}\right)\right),
$$

$\mathrm{e}$

$$
c_{m}:=-\log \left(\sup _{x} \nu\left(x_{0} \mid x_{-\infty}^{-1}\right)\right) .
$$

Segue de (4.3.3) que $0<c_{m} \leq c_{M}<1$. Então para todo $k$-cilìndro $\left[a_{1}^{k}\right] \in$ $A^{\mathbb{Z}}, k \geq 1$ vale

$$
e^{-c_{M} k} \leq \nu\left(a_{1}^{k}\right) \leq e^{-c_{m} k}
$$

Isto prova que $e_{k}$ é estritamente positiva e uniformemente limitada. Como consêquencia de (2.3.1) podemos concluir a prova.

Lema 4.3.2. Se $\left(X_{n}\right)_{n \in \mathbb{Z}}$ for uma c.o.i.p.l.c.e. então para todo $d \geq 0$, para todo $i, j \geq 1$ e para todo $k \geq 1$ valem as seguintes propriedades

1)

$$
\left|C_{\phi}(d)\right|=O\left(\gamma_{d}^{*}\right), \quad\left|C_{\phi_{i}}(d)\right|=O\left(\gamma_{d}^{*}\right), \quad \text { e }\left|C_{\phi_{i}, \phi_{j}}(d)\right|=O\left(\gamma_{d}^{*}\right)
$$


2) existe $M$ tal que

$$
\left|C_{\phi_{i}, \phi_{j}}(d)-C_{\phi}(d)\right| \leq M \gamma_{\text {inf }\{i, j\}}
$$

3) existe $M^{\prime}$ tal que

$$
\left|C_{\phi_{i}}(d)-C_{\phi}(d)\right| \leq M^{\prime} \gamma_{\sup \{i, d\}}^{*} ;
$$

4)

$$
\left|C_{I\left[a_{1}^{k}\right]}(d)\right| \leq 2 \nu\left(\left[a_{1}^{k}\right]\right) e^{-c_{m} \inf \{k, d\}} .
$$

5) existe $M^{\prime \prime}$ tal que

$$
\left|C_{I\left[a_{1}^{k}\right]}(d)\right| \leq M^{\prime \prime} \nu\left(\left[a_{1}^{k}\right]\right) \frac{\gamma_{d}^{*}}{\gamma_{k}} .
$$

Lembramos que se $\gamma_{k}=\exp ^{-c k}$ então $\gamma_{k}^{*}=\exp -c^{\prime} k$ onde $c^{\prime}<c$.

Prova. Observamos que (4.3.4) é consequência do Teorema 2.3.3. A partir da Definição 2.3.2, usando (3.1.7), (3.1.5) e o Lema 4.3.1 temos

$$
\begin{aligned}
\left|C_{\phi_{i}, \phi_{j}}(d)-C_{\phi}(d)\right| & \leq 2\left(\left\|\phi_{i}-\phi\right\|_{\infty} h+\left\|\phi_{j}-\phi\right\|_{\infty} h_{i}\right) \\
& \leq M \gamma_{\text {inf }\{i, j\}} .
\end{aligned}
$$

Fazendo $j=i$ em (4.3.5) e por outro lado usando (4.3.4) podemos concluir (4.3.6).

Como jà foi provado no Lema 4.3.1, temos que existem duas constantes $0<$ $c_{m} \leq c_{M}<1$ tais que para todo $k$-cilìndro $\left[a_{1}^{k}\right] \in A^{\mathbb{Z}}, k \geq 1$ vale

$$
e^{-c_{M} k} \leq \nu\left(a_{1}^{k}\right) \leq e^{-c_{m} k} .
$$

Como consequência do Teorema 2.3.3 e do Lema 4.3.1 (ii) segue (4.3.8).

Lema 4.3.3. Para qualquer sequência $(k(n))_{n \in \mathbb{N}}$ tal que $\lim _{n \rightarrow+\infty} k(n)=+\infty$ e para qualquer sequência $(s(n))_{n \in \mathbb{N}}$ tal que $\lim _{n \rightarrow+\infty} s(n)=+\infty$ vale

$$
\lim _{n \rightarrow+\infty}\left(\sigma_{\phi_{k(n)}}^{2}\right)^{s(n)}=\sigma_{\phi}^{2} .
$$


Prova. Segundo (2.3.4) e 2.3.5 temos

$$
\begin{aligned}
\left|\left(\sigma^{2}\right)_{\phi_{k(n)}}^{s(n)}-\sigma_{\phi}^{2}\right| \leq & \left|C_{\phi_{k(n)}}(0)-C_{\phi}(0)\right|+2 \sum_{d=1}^{s(n)-1}\left|C_{\phi_{k(n)}}(d)-C_{\phi}(d)\right| \\
& +2 \sum_{d=s(n)}^{+\infty}\left|C_{\phi}(d)\right|+\frac{2}{s(n)} \sum_{d=1}^{+\infty} d\left|C_{\phi_{k(n)}}(d)\right|:=A+B+C+D .
\end{aligned}
$$

Cada um desses termos serão analisados separadamente. Segundo (4.3.6) temos

$$
A:=\left|C_{\phi_{k(n)}}(0)-C_{\phi}(0)\right|=O\left(\gamma_{k(n)}^{*}\right)
$$

$\mathrm{e}$

$$
\begin{aligned}
B & :=2 \sum_{d=1}^{s(n)-1}\left|C_{\phi_{k(n)}}(d)-C_{\phi}(d)\right| \\
& \leq 2 \sum_{d=1}^{k(n)}\left|C_{\phi_{k(n)}}(d)-C_{\phi}(d)\right|+2 \sum_{d=k(n)+1}^{+\infty}\left|C_{\phi_{k(n)}}(d)-C_{\phi}(d)\right| \\
& =O\left(k(n) \gamma_{k(n)}^{*}\right)+O\left(\gamma_{k(n)}^{*}\right) .
\end{aligned}
$$

Segundo(4.3.4) temos

$$
C:=2 \sum_{d=s(n)}^{+\infty}\left|C_{\phi}(d)\right|=O\left(\gamma_{s(n)}^{*}\right)
$$

$\mathrm{e}$

$$
D:=\frac{2}{s(n)} \sum_{d=1}^{+\infty} d\left|C_{\phi_{k(n)}}(d)\right|=O\left(\frac{1}{s(n)}\right) .
$$

Como $k(n) \gamma_{k(n)}^{*}$ e $\gamma_{s(n)}^{*}$ convergem para zero podemos concluir a prova.

Lema 4.3.4. Para qualquer sequência $(k(n))_{n \in_{N}}$ tal que $\lim _{n \rightarrow+\infty} k(n)=+\infty$ vale

$$
\lim _{n \rightarrow \infty} \sup _{a \in A^{Z}}\left(\frac{\left(\sigma_{I\left[a_{1}^{k(n)}\right]}^{2}\right)^{n}}{\nu\left(a_{1}^{k(n)}\right)}\right)<+\infty .
$$


Prova. Segundo (4.3.7) e (2.3.4) temos

$$
\left(\sigma_{I\left[a_{1}^{k(n)}\right]}^{2}\right)^{n} \leq\left|C_{I\left[a_{1}^{k(n)}\right]}(0)\right|+2 \sum_{d=1}^{n-1}\left(1+\frac{d}{n}\right)\left|C_{I\left[a_{1}^{k(n)}\right]}(d)\right| .
$$

Para cada $n \in \mathbb{N}$ definimos $m(n)=k(n)+1+\left[e^{c_{m} k(n)}\right]$ e $i(n)=\inf \{m(n), n\}$. No membro direito da precedente desigualdade escrevemos o segundo termo como a soma de tres termos $A+B+C$. Usando (4.3.7) temos

$$
\begin{gathered}
\left|C_{I\left[a_{1}^{k(n)}\right]}(0)\right| \leq 2 \nu\left(\left[a_{1}^{k(n)}\right]\right), \\
A:=2 \sum_{d=1}^{k(n)}\left(1+\frac{d}{n}\right)\left|C_{I\left[a_{1}^{k(n)}\right]}(d)\right| \\
\leq 4 \nu\left(\left[a_{1}^{k(n)}\right]\right) \sum_{d=1}^{k(n)}\left(1+\frac{d}{n}\right) e^{-c_{m} d},
\end{gathered}
$$

e

$$
\begin{aligned}
B & :=2 \sum_{d=k(n)+1}^{i(n)-2}\left(1+\frac{d}{n}\right)\left|C_{I\left[a_{1}^{k(n)}\right]}(d)\right| \\
& \leq 8 \nu\left(\left[a_{1}^{k(n)}\right]\right) e^{-c_{m}(k(n)+1)}(i(n)-k(n)-1) .
\end{aligned}
$$

Usando (4.3.8) temos

$$
\begin{aligned}
C & :=2 \sum_{d=i(n)-1}^{n-1}\left(1+\frac{d}{n}\right)\left|C_{I\left[a_{1}^{k(n)}\right]}(d)\right| \\
& \leq M^{\prime \prime} \nu\left(\left[a_{1}^{k(n)}\right]\right) \sum_{d=i(n)-1}^{n-1} e^{c k(n)-c^{\prime} d} .
\end{aligned}
$$

Isto conclui a prova do lema.

Lema 4.3.5. Para qualquer sequências $(k(n))_{n \geq 1} e(s(n))_{n \geq 1}$ tal que $\lim _{n \rightarrow \infty} k(n)=$ $+\infty$ e $\lim _{n \rightarrow+\infty} \frac{k(n)}{s(n)}=0$ vale

$$
\lim _{n \rightarrow \infty}\left(\sigma_{e_{k(n)}}^{2}\right)^{s(n)}=\sigma_{\phi}^{2}
$$


Prova. Observamos a partir das definições de $\phi_{k}$ e $e_{k}$ (ver (3.1.3)) que

$$
e_{k}=\frac{1}{k} \sum_{d=1}^{k} T_{d} \phi_{d-1} .
$$

Enão usando a Definição 2.3.2 temos

$$
\begin{aligned}
C_{e_{k}}(d) & =\frac{1}{k^{2}}\left[\int \sum_{i, j=1}^{k} T_{i} \phi_{i-1} T_{(d+j)} \phi_{j-1}-\left(\int \sum_{i=1}^{k} T_{i} \phi_{i-1}\right)^{2}\right] \\
& =\frac{1}{k^{2}} \sum_{i, j=1}^{k} C_{\phi_{i-1}, \phi_{j-1}}(d+j-i) .
\end{aligned}
$$

Então

$$
\begin{aligned}
\left(\sigma_{e_{k(n)}}^{2}\right)^{s(n)} & =C_{e_{k(n)}}(0)+2 \sum_{d=1}^{s(n)-1} C_{e_{k(n)}}(d)-\frac{2}{s(n)} \sum_{d=1}^{s(n)-1} d C_{e_{k(n)}}(d) \\
& =\frac{1}{k^{2}(n)} \sum_{i, j=1}^{k(n)} C_{\phi_{i-1}, \phi_{j-1}}(j-i) \\
& +2 \sum_{d=1}^{s(n)-1} \frac{1}{k^{2}(n)} \sum_{i, j=1}^{k(n)} C_{\phi_{i-1}, \phi_{j-1}}(d+j-i) \\
& -\frac{2}{s(n)} \sum_{d=1}^{s(n)-1} \frac{1}{k^{2}(n)} \sum_{i, j=1}^{k(n)} d C_{\phi_{i-1}, \phi_{j-1}}(d+j-i)
\end{aligned}
$$

Observaremos que (4.3.4) e (4.3.5) continuam verdadeiras se no lugar de $C_{\phi_{i}, \phi_{j}}(d)$ colocamos $C_{l}(d)$ onde $l=\inf \{i, j\}$. Por essa razão denotaremos por $\left(\tilde{\sigma}_{e_{k(n)}}^{2}\right)^{s(n)}$ a expressão precedente (4.3.9) onde $C_{\phi_{i}, \phi_{j}}(d)$ será trocado por $C_{l}(d) \operatorname{com} l=$ $\inf \{i, j\}$. Em (4.3.9) agrouparemos juntos no termo $\mathcal{T}_{n}(d)$ as covariâncias do mesmo espaçamento $d$. Isto é

$$
\left(\tilde{\sigma}_{e_{k(n)}^{2}}^{2}\right)^{s(n)}=\sum_{d=0}^{s(n)+k(n)-2} \mathcal{T}_{n}(d) .
$$


Enão

$$
\begin{aligned}
\left|\left(\tilde{\sigma}_{e_{k(n)}}^{2}\right)^{s(n)}-\sigma_{\phi}^{2}\right| \leq & \left|\mathcal{T}_{n}(0)-C_{\phi}(0)\right|+\sum_{d=1}^{k(n)}\left|\mathcal{T}_{n}(d)-2 C_{\phi}(d)\right| \\
& +\sum_{d=k(n)}^{s(n)+k(n)-2}\left|\mathcal{T}_{n}(d)\right|+2 \sum_{d=k(n)}^{+\infty}\left|C_{\phi}(d)\right| .
\end{aligned}
$$

Nas seguintes cotas usaremos

$$
C_{\phi_{i}, \phi_{j}}(-d)=C_{\phi_{j}, \phi_{i}}(d) .
$$

Escreveremos o membro direito de (4.3.11) como uma soma de quatro termos $A+B+C+D$.

- Em primeiro vamos estudar o termo $\mathcal{T}_{n}(0)$.

$$
\mathcal{T}_{n}(0)=\frac{1}{k^{2}(n)} \sum_{i=1}^{k(n)}[2(k(n)-i)+1] C_{i-1}(0)-\mathcal{T}_{n}^{1}(0) .
$$

onde

$$
\mathcal{T}_{n}^{1}(0)=\frac{1}{s(n) k^{2}(n)} \sum_{i=1}^{k(n)}[k(n)-i][k(n)-i+1] C_{i-1}(0)=O\left(\frac{k(n)}{s(n)}\right)
$$

Usando (4.3.6) temos

$$
\begin{aligned}
A: & =\left|\mathcal{T}_{n}(0)-C_{\phi}(0)\right| \\
& =\frac{1}{k^{2}(n)} \sum_{i=1}^{k(n)}[2(k(n)-i)+1]\left|C_{i-1}(0)-C_{\phi}(0)\right|+O\left(\frac{k(n)}{s(n)}\right) \\
& \leq \frac{M^{\prime}}{k^{2}(n)} \sum_{i=1}^{k(n)}[2(k(n)-i)+1] \gamma_{i-1}+O\left(\frac{k(n)}{s(n)}\right) \\
& \leq O\left(\frac{1}{k(n)}\right)+O\left(\frac{k(n)}{s(n)}\right) .
\end{aligned}
$$

Então

$$
\lim _{n \rightarrow+\infty}\left|\mathcal{T}_{n}(0)-C_{\phi}(0)\right|=0 .
$$


- Para cada $1 \leq d \leq k(n)-1$

$$
\mathcal{T}_{n}(d)=\frac{2}{k^{2}(n)} \sum_{i=1}^{k(n)}[2(k(n)-i)+1] C_{i-1}(d)+\mathcal{T}_{n}^{1}(d) .
$$

onde

$$
\begin{aligned}
\mathcal{T}_{n}^{l}(d) & =\frac{2}{s(n) k^{2}(n)} \sum_{i=1}^{k(n)-d-1}\left[(k(n)-i+1)(k(n)-i)+d^{2}\right] C_{i-1}(d) \\
& +\frac{2}{s(n) k^{2}(n)} \sum_{i=k(n)-d}^{k(n)}[2(k(n)-i)+1] C_{i-1}(d) .
\end{aligned}
$$

Usando (4.3.4) temos

$$
\mathcal{T}_{n}^{1}(d)=O\left(\frac{k(n)}{s(n)}\right) \gamma_{d}^{*}
$$

Para estudar o segundo termo $B$ usaremos (4.3.6).

$$
\begin{aligned}
B_{n}(d): & =\left|\mathcal{T}_{n}(d)-2 C_{\phi}(d)\right| \\
& \leq \frac{2}{k^{2}(n)} \sum_{i=1}^{k(n)}[2(k(n)-i)+1] \| C_{i-1}(d)-C_{\phi}(d) \mid \\
& +O\left(\frac{k(n)}{s(n)}\right) \gamma_{d}^{*} \\
& \leq \frac{M^{\prime}}{k^{2}(n)} \sum_{i=1}^{k(n)}[2(k(n)-i)+1] \gamma_{\sup \{i-1, d\}}+O\left(\frac{k(n)}{s(n)}\right) \gamma_{d}^{*} \\
& =O\left(\frac{1}{k(n)}\right) d \gamma_{d}+O\left(\frac{1}{k(n)}\right) \gamma_{d}+O\left(\frac{k(n)}{s(n)}\right) \gamma_{d}^{*} .
\end{aligned}
$$

Então

$$
B:=\sum_{d=1}^{k(n)} B_{n}(d)=O\left(\frac{1}{k(n)}\right) .
$$

- Como consêquencia de (4.3.9) temos para cada $d \geq k(n)$

$$
\left|\mathcal{T}_{n}(d)\right| \leq \frac{2}{k^{2}(n)}\left(1+\frac{d}{n}\right) \sum_{i=1}^{k(n)}[2(k(n)-i)+1]\left|C_{i-1}(d)\right| .
$$


Então usando (4.3.4) temos

$$
\left|\mathcal{T}_{n}(d)\right|=O(1) \gamma_{d}^{*}
$$

Isto implica

$$
C:=\sum_{d=k(n)}^{s(n)+k(n)-2}\left|\mathcal{T}_{n}(d)\right|=O\left(\gamma_{k(n)}^{*}\right) .
$$

Finalmente usando (4.3.6) temos

$$
D:=\sum_{d=k(n)}^{+\infty}\left|C_{\phi}(d)\right|=O\left(\gamma_{k(n)}^{*}\right) .
$$

Como todos os termos A, B, C e D convergem para zero, o lema esta provado.

\subsection{Prova do Teorema-limite central}

Nesta Seção apresentaremos uma sequência de Lemas que provam o Teorema 4.1.1. Usaremos as notações e as definições introduzidas na Seção 4.1.

Lema 4.4.1. 1) Se $k(n)=o(s(n))$ então para cada $m \geq 1$ vale

$$
\mathrm{E}\left|Q_{(\bar{N}(n), k(n))}\right|^{m}=O\left(s^{m}(n)\right) .
$$

2) $\operatorname{Se} s(n)=o(\sqrt{n})$ então

$$
\frac{Q_{(\tilde{N}(n), k(n))}}{\sqrt{n}} \stackrel{\mathrm{P}}{\rightarrow} 0 .
$$

Prova. Segue de (4.1.7) e do fato que as funções $f_{k(n)}$ são uniformementes limitadas, que existe uma constante $M$ tal que

$$
\begin{aligned}
\left|Q_{(\bar{N}(n), k(n))}\right| & \leq M\left(\tilde{\tau}_{\bar{N}(n)+1, n}-n+\tilde{\tau}_{0, n}+2(k(n)-1)\right) \\
& \leq M\left(\Delta_{\tilde{\tau}_{\tilde{N}(n)+1, n}}+\tilde{\tau}_{0, n}+2(k(n)-1)\right) .
\end{aligned}
$$


Provaremos que $\mathbf{E}\left|Q_{(\tilde{N}(n), k(n))}\right|=O(s(n))$. Observamos que

$$
\mathrm{E} \Delta_{\tilde{\tau}_{\tilde{N}(n)+1, n}}=\mathbf{E}\left(\mathbf{E}\left(\Delta_{\tilde{\tau}_{\tilde{N}(n)+1, n}} \mid \tilde{N}(n)+1\right)\right) .
$$

Por outro lado, $\{j \leq \tilde{N}(n)+1\}=\left\{\tilde{\tau}_{j-1, n}>n\right\}^{c}$. Então o evento $\{j=$ $\tilde{N}(n)+1\}$ pode ser escrito em função das variáveis aleatórias $\Delta_{\bar{\tau}_{i, n}} \operatorname{com} i \leq j-1$. Então, como a sequência $\left(\triangle_{\tilde{\tau}_{j, n}}\right)_{j \geq 0}$ é constituida de variáveis aleatórias independentes (ver o Lema 4.2.5), temos que $\{j=\tilde{N}(n)+1\}$ é independente $\Delta_{\tilde{\tau}_{j, n}}$. Então segue de (4.4.1)que

$$
\mathrm{E} \triangle_{\tilde{\tau}_{\tilde{N}(n)+1, n}}=\mathbf{E} \triangle_{\tilde{\tau}_{0, n}}
$$

Usando (4.2.10) temos que $\mathbf{E} \Delta_{\tilde{\tau}_{\tilde{N}(n)+1, n}}=O(s(n))$ e como $\mathbf{E} \tilde{\tau}_{0, n}<+\infty$ podemos concluir a prova do Lema usando a desigualdade de Chebychev .

Denotaremos as variáveis aleatórias $R_{i}^{\prime}$ definidas em (4.1.5) por $R^{\prime}\left(\tilde{\tau}_{i, n}\right)=R_{i}^{\prime}$. Definiremos uma nova sequência de variáveis aleatórias $R^{\prime}\left(\tau_{i}\right)$ como sendo

$$
R^{\prime}\left(\tau_{i}\right):=\sum_{j=\tau_{i}-k}^{\tau_{i}-1}\left[T_{(j+k(n))} f_{k(n)}-\mathbf{E}_{\nu}\left[f_{k(n)}\right]\right] .
$$

Lema 4.4.2. Seja $\left(s(n)_{n \geq 1}\right)$ uma sequência tal que $s(n) \geq 2 k(n)$ para todo $n \geq$ 1. Então

1)para cada $n \geq 1$, as sequências $\left(R_{i}^{\prime}\right)_{i \geq 0} e\left(R_{i}\right)_{i \geq 0}$, definidas em (4.1.5) $e$ respectivamente em (4.1.10), são constituídas de variáveis aleatórias i.i.d.

2) para cada $l \geq 1$ temos que a variável aleatória $R^{\prime}\left(\tau_{l}\right)$, definida em (4.4.2), é independente de $\Delta_{\tau_{j}}$ para qualquer $j \leq l-1$.

3) para cada $i \geq 1$ temos que a variável aleatória $R_{i}^{\prime}$ é independente de $\Delta_{\tilde{\tau}_{j}}$ para qualquer $j \leq i-1$.

4) para todo $i \geq 1$ as variáveis aleatórias $1(i \leq \tilde{N}(n)+1)$ e $R_{i}^{\prime}$ são independentes.

5) valem as seguintes igualdades

$$
\begin{aligned}
& \mathbf{E}\left(\sum_{i=1}^{\tilde{N}(n)+1} R_{i}^{\prime}\right)=\mathrm{E}(\tilde{N}(n)+1) \mathrm{E} R_{1}^{\prime} \\
& \mathbf{E}\left(\sum_{i=1}^{\bar{N}(n)+1} R_{i}\right)^{2}=\mathrm{E}(\tilde{N}(n)+1) \mathrm{E} R_{1}^{2}
\end{aligned}
$$


Prova. 1) Segue de (4.1.5) que $R_{i}^{\prime}=R_{i}^{\prime}\left(X_{\tilde{\tau}_{i}-k(n)}^{\tilde{\tau}_{i}+k(n)-1}\right)$. Então, como consequência do Corolário 2.2.11, $\left(R_{i}^{\prime}\right)_{i \geq 1}$ é uma sequência que contém variáveis aleatórias i.i.d. Pela mesma razão $R_{i}$ é uma sequência que também é constituida de variáveis aleatórias i.i.d..

2)Observaremos que $R^{\prime}\left(\tau_{l}\right)$ é uma função de $X_{\tau_{l-1}}^{+\infty}$ então, como consequência do Lema 4.2 .4 temos que $R^{\prime}\left(\tau_{l}\right)$ é independente de $\triangle_{\tau_{j}}$ para todo $j \leq l-1$.

3) Como $\tilde{\tau}_{i, n}:=\tau_{s(n) i}$ temos $R_{i}^{\prime}=R^{\prime}\left(\tilde{\tau}_{i, n}\right)=R^{\prime}\left(\tau_{s(n) i}\right)$. Segue do item 2 do Lema que $R^{\prime}\left(\tilde{\tau}_{i, n}\right)$ é independente de $\Delta_{\tau_{j}}$ para todo $j \leq s(n) i-1$. Observamos por outro lado que $\Delta_{\bar{\tau}_{j, n}}=\sum_{l=0}^{s(n)-1} \Delta_{\tau_{s(n) j-l}}$.

Isto conclui a prova do ítem 3 do Lema.

4) Observamos que o evento $\{i \leq \tilde{N}(n)+1\}=\left\{\tilde{\tau}_{i-1, n}>n\right\}^{c}$. Então as variaveis $1(i \leq \tilde{N}(n)+1)$ podem ser escritas em função das variáveis aleatórias $\Delta_{\tilde{\tau}_{j, n}} \operatorname{com} j \leq i-1$. Usando o ítem 3 do Lema concluimos que as variáveis aleatórias $1(i \leq \tilde{N}(n)+1)$ e $R_{(i, k(n), n)}^{\prime}$ são independêntes para todo $i \geq 1$.

5) Como consequência do item 4 temos

$$
\begin{aligned}
\mathrm{E}\left(\sum_{i=1}^{\bar{N}(n)+1} R_{i}^{\prime}\right) & =\sum_{i=1}^{\infty} \mathbf{E}\left(1(i \leq \tilde{N}(n)+1) R_{i}^{\prime}\right) \\
& =\sum_{i=1}^{\infty} \mathbf{E} 1(i \leq \tilde{N}(n)+1) \mathbf{E} R_{i}^{\prime} \\
& =\mathbf{E}(\tilde{N}(n)+1) \mathbf{E} R_{1}^{\prime}
\end{aligned}
$$

Por outro lado temos

$$
\begin{aligned}
\mathrm{E}\left(\sum_{i=1}^{\bar{N}(n)+1} R_{i}\right)^{2}= & \sum_{i=1}^{\infty} \mathrm{E}\left(1(i \leq \tilde{N}(n)+1) R_{i}^{2}\right) \\
& +2 \sum_{1 \leq i<j}^{\infty} \mathrm{E}\left(1(j \leq \tilde{N}(n)+1) R_{i} R_{j}\right) \\
= & \sum_{i=1}^{\infty} \mathrm{E} 1(i \leq \tilde{N}(n)+1) \mathrm{E} R_{i}^{2} \\
= & \mathrm{E}(\tilde{N}(n)+1) \mathrm{E} R_{1}^{2} .
\end{aligned}
$$

Pois de um lado, para cada $j>i$ as variáveis aleatórias $R_{j}^{\prime}$ são independentes de $1(j \leq \tilde{N}(n)+1) R_{i}$ e por outro lado. temos $\mathrm{E} R_{j}=0$. Isto conclui a prova do 
Lema.

Lema 4.4.3. Se $\left(k(n)_{n \geq 1}\right)$ for uma sequência tal que $\lim _{n \rightarrow \infty} k(n)=+\infty$ então para cada $m \geq 1$ valem

$$
\begin{aligned}
\mathbf{E}\left|R_{1}^{\prime}\right|^{m} & =O\left(k^{m}(n)\right) \\
\mathrm{E}\left|R_{1}\right|^{m} & =O\left(k^{m}(n)\right) .
\end{aligned}
$$

Em particular se $f_{k}=\phi_{k}$, definida em (3.1.3), vale

$$
\begin{array}{r}
\left|\mathbf{E} R_{1}^{\prime}\right|<+\infty . \\
\mathbf{E}\left[\left(R_{1}\right)^{2}\right]=O(k(n)) .
\end{array}
$$

Prova. Observamos que

$$
\begin{aligned}
R_{1}^{\prime} & :=\sum_{j=\widetilde{\tau}_{1, n}-k(n)}^{\tilde{\tau}_{1, n}-1}\left[T_{(j+k(n))} f_{k(n)}-\mathbf{E}_{\nu}\left(f_{k(n)}\right)\right] \\
& =\sum_{j=0}^{k(n)-1}\left[T_{\left(\widetilde{\tau}_{1, n}+j\right)}\left(f_{k(n)}\right)-\mathbf{E}_{\nu}\left(f_{k(n)}\right)\right]
\end{aligned}
$$

Então, como as funções $f_{k(n)}$ são uniformemente limitadas, podemos concluir (4.4.5). Para cada $j \geq 1$ definimos

$$
D_{j}(f):=\mathbf{E}\left(T_{\tau_{0}+j} f\right)-\mathbf{E}(f) .
$$

Escreveremos (4.4.7) como

$$
\mathrm{E} R_{1}^{\prime}=\sum_{j=0}^{k(n)-1} D_{j}\left(f_{k(n)}\right) .
$$

Segue do Lema 4.3.1 ítem(ii), que $\operatorname{var}_{j}\left(\phi_{k(n)}\right) \leq \gamma_{j}$. Isto prova que $\sum_{j=1}^{\infty} j \operatorname{var}_{j}\left(\phi_{k}\right)<$ $+\infty$. Então em consequência do Lema 4.2.7 ítem (ii) temos $\left|\mathbf{E} R_{1}^{\prime}\left(\phi_{k}\right)\right|<+\infty$.

Por outro lado, usando o Corolário 4.2.8 temos

$$
\mathrm{E}\left[\left(R_{1}^{\prime}\right)^{2}\right]-k(n)\left(\sigma_{\phi_{k(n)}}^{2}\right)^{k(n)}=O(k(n)) .
$$

Então como $\left(\sigma_{\phi_{k(n)}}^{2}\right)^{k(n)}<+\infty$ temos $\mathbf{E}\left[\left(R_{1}^{\prime}\right)^{2}\right]=O(k(n))$. 
Para concluir o lema so precisamos observar que

$$
\mathbf{E}\left[\left(R_{1}\right)^{2}\right]=\mathbf{E}\left[\left(R_{1}^{\prime}\right)^{2}\right]-\left(\mathbf{E} R_{1}^{\prime}\right)^{2} .
$$

Lema 4.4.4. Se $k^{2}(n)=o(s(n))$ então

$$
\frac{\sum_{i=1}^{\bar{N}(n)} R_{(i, k(n), n)}}{\sqrt{n}} \stackrel{\mathrm{P}}{\rightarrow} 0 .
$$

Prova. Usando (4.4.4), (4.4.5) e (4.2.15) temos

$$
\begin{aligned}
\mathbf{E}\left(\left[\sum_{i=1}^{\bar{N}(n)+1} R_{1}\right]^{2}\right) & =\mathbf{E}(\tilde{N}(n)) O\left(k^{2}(n)\right) \\
& =O\left(\frac{n k^{2}(n)}{s(n)}\right) .
\end{aligned}
$$

Para concluir a prova do lema usamos a desigualdade de Chebychev de ordem dois.

Lema 4.4.5. Seja $(s(n))_{n \geq 1}$ uma sequência de inteiros tal que $s(n)>k(n)+$ 1. Para s variáveis aleatórias $S_{i}^{\prime}$ definidas em (4.1.4) temos as seguintes propriedades:

1) para cada $n \geq 1$, as sequências ${ }^{\wedge}\left(S_{i}^{\prime}\right)_{i \geq 0} e\left(S_{i}\right)_{i \geq 0}$ são constituídas de variáveis aleatórias i.i.d..

2) valem as seguintes igualdades

$$
\begin{aligned}
\mathrm{E}\left(\sum_{i=0}^{\bar{N}(n)} S_{i}^{\prime}\right) & =\mathrm{E}(\tilde{N}(n)+1) \mathbf{E} S_{0}^{\prime} \\
\mathrm{E}\left(\sum_{i=0}^{\tilde{N}(n)} S_{i}\right)^{2} & =\mathrm{E}(\tilde{N}(n)+1) \mathrm{E} S_{0}^{2}
\end{aligned}
$$


Prova. 1)Segue de (4.1.4) que $S_{i}^{\prime}=S_{i}^{\prime}\left(X_{\tilde{\tau}_{i, n}}^{\tilde{\tau}_{i+1, n}-1}\right)$. Então, como consequência do Corolario 2.2.11, concluimos o primeiro ítem do Lema.

2) Temos $\{i \leq \tilde{N}(n)\}=\left\{\tilde{\tau}_{i, n}>n\right\}^{c}$. Então as variáveis aleatórias $\mathbf{1}(i \leq$ $\tilde{N}(n))$ podem ser escritas em função de $\Delta_{\tilde{\tau}_{j, n}} \operatorname{com} j \leq i$. Observaremos que $\overline{S_{i}^{\prime}}$ é uma função de $X_{\tau_{s(n) i}}^{\infty}$ então, como consequência do Lema 4.2.4 temos que $S_{i}^{\prime}$ é independente de $\Delta_{\tau_{j}}$ para todo $j \leq s(n) i$. Como $\Delta_{\tilde{\tau}_{j, n}}=\sum_{l=0}^{s(n)-1} \Delta_{\tau_{s(n) j-l}}$ então $S_{i}^{\prime}$ é independente de $\Delta_{\tilde{\tau}_{j, n}}$ para todo $j \leq i$. Concluimos a prova do item 2 do lema fazendo referência á prova do (4.4.3) e (4.4.4).

Lema 4.4.6. Valem as seguintes propriedades:

1)

$$
\mathbf{E} S_{1}^{\prime}+\mathbf{E} R_{1}^{\prime}=-\frac{\mathbf{E} Q_{(\bar{N}(n), k(n))}}{\mathbf{E}(\tilde{N}(n)+1)}
$$

e consequientemente

$$
L_{(\bar{N}(n), k(n))}=-(\tilde{N}(n)+1) \frac{\mathbf{E} Q_{(\tilde{N}(n), k(n))}}{\mathbf{E}(\tilde{N}(n)+1)} .
$$

2) $\operatorname{Se} s(n)=o(\sqrt{n})$ ent $\tilde{a} o$

$$
\frac{L_{(\bar{N}(n), k(n))}}{\sqrt{n}} \stackrel{\mathrm{P}}{\rightarrow} 0 .
$$

$e$

$$
\mathbf{E} L_{(\bar{N}(n), k(n))}^{2}=O\left(s^{4}(n)\right) .
$$

Prova. 1) Calcularemos a esperança de cada membro da igualdade (4.1.8). Em consequência de (4.4.9) e (4.4.3) temos

$$
0=\mathbf{E}(\tilde{N}(n)+1) \mathbf{E}\left(S_{1}^{\prime}\right)+\mathbf{E}(\tilde{N}(n)+1) \mathbf{E}\left(R_{1}^{\prime}\right)+\mathbf{E}\left(Q_{(\bar{N}(n), k(n))}\right) .
$$

Isto conclui a prova do (4.4.11).

Como consequência de (4.4.11) e de (4.1.12) concluimos (4.4.12).

2) Segue do (4.4.12) e do Lema 4.4.1 que $\mathrm{E} L_{(\bar{N}(n), k(n))}=O(s(n))$. Então usando a desigualdade de Chebychev concluimos a prova. 
3) Como consequência de (4.4.12), (4.4.6), (4.2.15) do Lema 4.4.1 e do fato que $N(n) \leq n$ temos

$$
\begin{aligned}
\mathrm{E} L_{(\tilde{N}(n), k(n))}^{2} & =\mathbf{E}\left((\tilde{N}(n)+1)^{2}\right)\left(\frac{\mathbf{E}\left(Q_{(\tilde{N}(n), k(n))}\right)}{\mathrm{E}(\tilde{N}(n)+1)}\right)^{2} \\
& =O\left(s^{4}(n)\right) .
\end{aligned}
$$

Lema 4.4.7. Seja $n \geq 1$. Para todo $m \geq 1$ valem

$$
\begin{array}{r}
\mathrm{E}\left|S_{1}^{\prime}\right|^{m}=O\left(s^{m}(n)\right) \\
\mathrm{E}\left|S_{1}\right|^{m}=O\left(s^{m}(n)\right) \\
\left|\mathbf{E} S_{1}^{\prime}\right|=O(k(n)) \\
\mathbf{E}{S^{\prime}}_{1}^{2}=O(s(n)) ; \\
\mathbf{E} S_{1}^{2}=O(s(n)) .
\end{array}
$$

Prova. Segue de (4.1.4) e do fato que as funções $f_{k}(n)$ são uniformemente limitadas, que existe uma constante $M$ tal que

$$
\left|S_{1}^{\prime}\right| \leq M\left|\Delta_{\tilde{\tau}_{1, n}}-k(n)\right|
$$

Isto prova (4.4.14) e usando (4.1.9) podemos concluir a prova de (4.4.15).

O resultado (4.4.16) segue de (4.4.11), de (4.4.5) e de (4.2.15) .

Para provar (4.4.17) escreveremos

$$
\begin{aligned}
S_{1}^{\prime} & :=\sum_{j=\tilde{\tau}_{1, n}}^{\bar{\tau}_{2, n}-k(n)-1}\left[T_{(j+k(n))} f_{k(n)}-\mathbf{E}_{\nu}\left(f_{k(n)}\right)\right] \\
& =\sum_{j=0}^{\Delta \tilde{\tau}_{1, n}-k(n)}\left(T_{\bar{\tau}_{1, n}+j+k(n)} f_{k(n)}-\mathbf{E}\left(f_{k(n)}\right)\right) \\
& =\sum_{j=0}^{\mathbf{E} \Delta \bar{\tau}_{1, n}-k(n)}\left(T_{\bar{\tau}_{1, n}+j+k(n)} f_{k(n)}-\mathbf{E}\left(f_{k(n)}\right)\right)+S_{1}^{\prime \prime}
\end{aligned}
$$


onde

$$
\begin{aligned}
S_{1}^{\prime \prime} & :=\sum_{j=0}^{\Delta \bar{\tau}_{1, n}-k(n)}\left(T_{\bar{\tau}_{1, n}+j+k(n)} f_{k(n)}-\mathbf{E}\left(f_{k(n)}\right)\right) \\
& -\sum_{j=0}^{\mathbf{E} \Delta \bar{\tau}_{1, n}-k(n)}\left(T_{\bar{\tau}_{1, n}+j+k(n)} f_{k(n)}-\mathbf{E}\left(f_{k(n)}\right)\right) .
\end{aligned}
$$

Observamos que

$$
\left|S_{1}^{\prime \prime}\right| \leq M\left|\Delta \tilde{\tau}_{1, n}-\mathbf{E} \Delta \tilde{\tau}_{1, n}\right| .
$$

Como consêquencia de (4.2.11) temos que a variança das variáveis $\Delta \tilde{\tau}_{i, n}$ é de ordem de $s(n)$. Então

$$
\mathbf{E}{S_{1}^{\prime \prime 2}}^{2}=O(s(n))
$$

Usando a notação $\left(\sigma_{f}\right)^{m}$, introduzida em (2.3.3), consideraremos

$$
\begin{aligned}
A & :=\mathbf{E}\left(\sum_{j=0}^{\mathrm{E} \Delta \tilde{\tau}_{1}-k(n)}\left(T_{\tilde{\tau}_{1, n}+j+k(n)} f_{k(n)}-\mathbf{E} f_{k(n)}\right)\right)^{2} \\
& -\left(\mathbf{E} \Delta \tilde{\tau}_{1}-k(n)\right)\left(\sigma_{f_{k(n)}}^{2}\right)\left(\mathbf{E} \Delta \tilde{\tau}_{1}-k(n)\right)
\end{aligned}
$$

e

$$
B:=\left(\mathbf{E} \Delta \tilde{\tau}_{1}-k(n)\right)\left(\sigma_{f_{k(n)}}^{2}\right)^{\left(\mathrm{E} \Delta \tilde{\tau}_{1}-k(n)\right)} .
$$

Como consequência do Corolário 4.2 .8 aplicado para as funções $f_{k(n)}$ e para $m=\mathrm{E} \Delta \tilde{\tau}_{1}-k(n)$ temos $A=O(1)$. Por outro lado usando a hipótese (iii) sobre as funções $f_{k(n)}$ temos $B=O(s(n))$. Então

$$
\mathrm{E}\left(\sum_{j=0}^{\mathrm{E} \triangle \bar{\tau}_{1}-k(n)}\left(T_{\bar{\tau}_{1, n}+j+k(n)} f_{k(n)}-\mathbf{E} f_{k(n)}\right)\right)^{2}=A+B=O(s(n)) .
$$

Então a partir da igualdade (4.4.19) usando (4.4.20), (4.4.21) e a desigualdade de Cauchy-Schwarz podemos concluir a prova do (4.4.17).

Como $\mathbf{E}\left[\left(S_{1}\right)^{2}\right]=\mathbf{E}\left[\left(S_{1}^{\prime}\right)^{2}\right]-\left(\mathbf{E}\left[S_{1}^{\prime}\right]\right)^{2}$, então (4.4.18) é consequência de (4.4.16) e (4.4.17). 
Lembramos que o nosso objetivo agora é de provar que a variável aleatória $\sum_{i=0}^{\bar{N}(n)} S_{i}$ converge em distribuição para uma variável gaussiana. Por essa razão queremos estudar o comportamento assintótico da variança da variável aleatória $\sum_{i=0}^{\bar{N}(n)} S_{i}$. Como consequência dos lemas precedentes temos o seguinte resultado.

Proposição 4.4.8. Sejam $\gamma$ definido em (2.2.7) e o definido em (4.0.1). Se $\lim _{n \rightarrow \infty} k(n)=$ $+\infty, k^{2}(n)=o(s(n))$ e $s^{4}(n)=O(n)$ então

$$
\lim _{n \rightarrow+\infty} \frac{\mathrm{E} S_{1}^{2}}{\gamma s(n)}=\sigma^{2} .
$$

Prova. Cada termo da igualdade (4.1.11) sera elevado ao quadrado. Depois, será calculado o valor esperado de cada membro. Isto é,

$$
\begin{aligned}
n\left(\sigma_{f_{k(n)}}^{2}\right)^{n} & =\mathrm{E}\left(\sum_{i=0}^{\tilde{N}(n)} S_{i}\right)^{2}+\mathrm{E}\left(\sum_{i=1}^{\tilde{N}(n)+1} R_{i}\right)^{2} \\
& +\mathrm{E}\left(Q_{(\tilde{N}(n), k(n))}\right)^{2}+\mathrm{E}\left(L_{(\tilde{N}(n), k(n))}\right)^{2}+A
\end{aligned}
$$

onde

$$
\begin{aligned}
A: & =2 \mathbf{E}\left(\sum_{i=1}^{\bar{N}(n)+1} R_{i}\right)\left(\sum_{i=0}^{\bar{N}(n)} S_{i}\right) \\
& +2 \mathbf{E}_{\mu_{C}}\left(Q_{(\bar{N}(n), k(n))}\right)\left(\sum_{i=0}^{\tilde{N}(n)} S_{i}\right) \\
& +2 \mathbf{E}\left(Q_{(\bar{N}(n), k(n))}\right)\left(\sum_{i=1}^{\bar{N}(n)+1} R_{i}\right) . \\
& +2 \mathbf{E}\left(L_{(\bar{N}(n), k(n))}\right)\left(\sum_{i=0}^{\bar{N}(n)} S_{i}\right) . \\
& +2 \mathbf{E}\left(L_{(\bar{N}(n), k(n))}\right)\left(\sum_{i=0}^{\tilde{N}(n)+1} R_{i}\right) \\
& +2 \mathbf{E}\left(L_{(\bar{N}(n), k(n))} Q_{(\bar{N}(n), k(n))}\right) .
\end{aligned}
$$


Como consequência de (4.4.23) e usando (4.4.4) e (4.4.10) temos

$$
\begin{aligned}
B & :=\frac{\mathbf{E} \tilde{N}(n)+1}{n} \mathbf{E} S_{1}{ }^{2}-\left(\sigma_{f_{k(n)}}^{2}\right)^{n} \\
& =-\frac{\mathbf{E} \tilde{N}(n)+1}{n} \mathbf{E} R_{1}{ }^{2}-\frac{1}{n} \mathbf{E} Q_{(\tilde{N}(n), k(n))}^{2} \\
& -\frac{1}{n}\left(\mathbf{E} L_{(\tilde{N}(n), k(n))}^{2}+A\right) .
\end{aligned}
$$

Sabemos que $\mathrm{E} Q_{(\bar{N}(n), k(n))}^{2}=O\left(s^{2}(n)\right)$ ( ver o Lema 4.4.1) e que $\mathrm{E} R_{1}{ }^{2}=$ $O\left(k^{2}(n)\right)(\operatorname{ver}(4.4 .6))$. Por outro lado temos que $\mathbf{E} S_{1}{ }^{2}=O(s(n))(\operatorname{ver}(4.4 .18)) \mathrm{e}$ que $\mathrm{E} L_{(\tilde{N}(n), k(n))}^{2}=O\left(s^{4}(n)\right)$ (ver o Lema 4.4.12). Usaremos também o resultado (4.2.15) sobre a esperança da variável $\tilde{N}(n)$.

Então

$$
\frac{\mathbf{E}\left(L_{(\tilde{N}(n), k(n))}^{2}\right)}{n}=O\left(\frac{s^{4}(n)}{n}\right)
$$

$\mathrm{e}$

$$
\frac{1}{n} \mathbf{E} Q_{(\bar{N}(n), k(n))}^{2}=\left(\frac{k^{2}(n)}{s(n)}\right) .
$$

Usando a desigualdade de Cauchy-Schwarz, temos

$$
\begin{aligned}
& \frac{\left|\mathrm{E} Q_{(\bar{N}(n), k(n))}\left(\sum_{i=0}^{\bar{N}(n)} S_{(i, k(n), n)}\right)\right|}{n} \leq \frac{\sqrt{\mathbf{E} Q_{(\tilde{N}(n), k(n))}^{2}(\mathbf{E} \tilde{N}(n)+1) \mathbf{E} S_{1}{ }^{2}}}{n} \\
& =O\left(\frac{s(n)}{\sqrt{n}}\right) \text {. } \\
& \frac{\mathrm{E}\left|Q_{(\bar{N}(n), k(n))}\left(\sum_{i=1}^{\tilde{N}(n)+1} R_{(i, k(n), n)}\right)\right|}{n} \leq \frac{\sqrt{\mathbf{E} Q_{(\bar{N}(n), k(n))}^{2}(\mathbf{E} \tilde{N}(n)+1) \mathbf{E} R_{1}^{2}}}{n} \\
& =O\left(\sqrt{\frac{s^{2}(n)}{n} \frac{k^{2}(n)}{s(n)}}\right) \text {. } \\
& \begin{aligned}
\frac{\mathrm{E}\left|\left(\sum_{i=1}^{\bar{N}(n)+1} R_{(i, k(n), n)}\right)\left(\sum_{i=0}^{\bar{N}(n)} S_{(i, k(n), n)}\right)\right|}{n} & \leq \frac{\sqrt{(\mathbf{E}(\tilde{N}(n))+1)^{2} \mathbf{E} S_{1}^{2} \mathbf{E} R_{1}^{2}}}{n} \\
& =\left(\sqrt{\frac{k^{2}(n)}{s(n)}}\right) .
\end{aligned}
\end{aligned}
$$




$$
\begin{aligned}
& \frac{\mathrm{E}\left|L_{(\bar{N}(n), k(n))}\left(\sum_{i=0}^{\tilde{N}(n)} S_{(i, k(n), n)}\right)\right|}{n} \leq \frac{\sqrt{\mathbf{E} L_{(\bar{N}(n), k(n))}^{2}(\mathbf{E} \tilde{N}(n)+1) \mathbf{E} S_{1}^{2}}}{n} \\
&=O\left(\frac{s(n)}{\sqrt{n}}\right) . \\
& \frac{\mathbf{E}\left|L_{(\bar{N}(n), k(n))}\left(\sum_{i=1}^{\bar{N}(n)+1} R_{(i, k(n), n)}\right)\right|}{n} \leq \frac{\sqrt{\mathbf{E} L_{(\bar{N}(n), k(n))}^{2}(\mathbf{E} \tilde{N}(n)+1) \mathbf{E} R_{1}^{2}}}{n} \\
& \frac{\mathbf{E}\left|L_{(\tilde{N}(n), k(n))} Q_{(\bar{N}(n), k(n))}\right|}{n} \leq \frac{\sqrt{\mathbf{E} L_{(\tilde{N}(n), k(n))}^{2} \mathbf{E} Q_{(\bar{N}(n), k(n))}^{2}}}{n} \\
&=O\left(\frac{\left.s^{4}(n)\right)}{n} \frac{k^{2}(n)}{s(n)}\right) . \\
& n
\end{aligned}
$$

Então $A=o(n)$. Em conclusão, em (4.4.24), cada um de termos do membro direito converge para zero e usando de novo (4.2.15) temos

$$
\lim _{n \rightarrow \infty}\left(\frac{1}{\gamma s(n)} \mathbf{E} S_{1}{ }^{2}-\left(\sigma_{f_{k(n)}}^{2}\right)^{n}\right)=0 .
$$

Usando a hipótese (iii) sobre as funções $f_{k(n)}$ podemos concluir a prova.

Lema 4.4.9. Sejam $(k(n))_{n \geq 1} e(s(n))_{n \geq 1}$ sequências tal que $k^{2}(n)=o(s(n)) e$ $s^{4}(n)=O(n)$.

1) $\mathrm{Se} \sigma^{2}>0$ então

$$
\frac{\sum_{i=0}^{\mathrm{E}(\bar{N}(n))} S_{(i, k(n), n)}}{\sigma \sqrt{n}} \stackrel{\mathcal{D}}{\rightarrow} \mathcal{N}(0,1) .
$$

2) $S e \sigma^{2}=0$ então

$$
\frac{\sum_{i=0}^{\mathrm{E}(\tilde{N}(n))} S_{(i, k(n), n)}}{\sqrt{n}} \stackrel{\mathrm{P}}{\rightarrow} 0
$$


Prova. 1)Escreveremos o teorema de Berry-Esseen para as variáveis aleatórias i.i.d. de média zero, $S_{i}=S_{(i, k(n), n)}$. Isto é

$$
\sup _{x \in \mathbb{R}}\left|\mathbf{P}\left(\frac{\sum_{i=0}^{\mathrm{E}(\bar{N}(n))} S_{i}}{\sqrt{(\mathrm{E} \tilde{N}(n)+1) \mathrm{E} S_{1}^{2}}} \leq x\right)-\Phi(x)\right| \leq C_{B E} \frac{\mathrm{E}\left(\mid S_{1}^{3}\right)}{\left.\left(\mathrm{E} S_{1}{ }^{2}\right)\right)^{\frac{3}{2}} \sqrt{\mathrm{E}(\tilde{N}(n)+1)}} .
$$

onde $C_{B E}$ é uma constante e $\Phi(x)=\int_{-\infty}^{x}(2 \pi)^{-1 / 2} e^{-y^{2} / 2} d y$.

Usando (4.4.15) e (4.4.22) temos

$$
\sup _{x \in \mathbb{R}}\left|\mathbf{P}\left(\frac{\sum_{i=0}^{\mathbf{E}(\bar{N}(n))} S_{i}}{\sqrt{\mathbf{E}(\tilde{N}(n)+1) \mathbf{E}{S_{1}}^{2}}} \leq x\right)-\Phi(x)\right|=O\left(\frac{s^{2}(n)}{\sqrt{n}}\right) .
$$

Observamos que

$$
\frac{\sum_{i=0}^{\mathrm{E}(\tilde{N}(n))} S_{i}}{\sigma \sqrt{n}}=c_{n} \frac{\sum_{i=0}^{\mathrm{E}(\tilde{N}(n))} S_{i}}{\sqrt{\mathrm{E}\left(S_{1}^{2}\right) \mathrm{E}(\tilde{N}(n)+1)}}
$$

onde

$$
c_{n}=\sqrt{\frac{\mathbf{E}(\tilde{N}(n)+1) \mathbf{E} S_{1}^{2}}{n \sigma^{2}}} .
$$

Usando (4.4.22) temos $\lim _{n \rightarrow+\infty} c_{n}=1$. Isto conclui a prova.

Finalmente temos o seguinte resultado (proposto como exercício em Billingsley (1995))

Lema 4.4.10.

$$
\frac{\sum_{i=0}^{\bar{N}(n)} S_{(i, k(n), n)}}{\sigma \sqrt{n}} \stackrel{\mathcal{D}}{\rightarrow} \tilde{\mathcal{N}}(0,1)
$$

Prova. Seja $\delta>0$. Escreveremos

$$
\mathrm{P}\left(\left|\frac{\sum_{i=0}^{\tilde{N}(n)} S_{i}}{\sqrt{n}}-\frac{\sum_{i=0}^{\mathrm{E}(\tilde{N}(n))} S_{i}}{\sqrt{n}}\right|>\delta\right) \leq A+B
$$


Onde

$$
A:=\mathbf{P}\left(|\tilde{N}(n)-\mathbf{E}(\tilde{N}(n))|>\delta^{3} \frac{\beta n}{s(n)}\right) .
$$

$\mathrm{e}$

$$
B:=\mathrm{P}\left(\sup _{|j-\mathrm{E}(\bar{N}(n))| \leq \delta^{3} \frac{\beta n}{s(n)}} \frac{\left|\sum_{i=0}^{j} S_{i}-\sum_{i=0}^{\mathrm{E}(\bar{N}(n))} S_{i}\right|}{\sqrt{n}}>\delta\right)
$$

Usando Lema 4.2.6, o termo $A$ converge para zero.

Usando a desiqualdade de Kolmogorov , escreveremos

$$
\begin{aligned}
B & \leq 2 \frac{\mathrm{E}\left(\sum_{i=0}^{\delta^{3} \frac{\beta n}{s(n)}} S_{i}\right)^{2}}{\delta^{2} \sigma^{2} n} \\
& \leq 2 \delta \frac{\beta \mathbf{E} S_{1}^{2}}{s(n)}
\end{aligned}
$$

Usando (4.4.22), o termo $B$ converge em probabilidade para zero.

\subsection{Entropia relativa}

Lembramos que a entropia relativa foi definida em (3.1.6). Como consequência do Lema 4.3.4 temos do seguinte resultado.

Teorema 4.5.1. Seja $\epsilon>0$. Para qualquer sequência $\left(k(n)_{n \in \mathbb{N}}\right)$ tal que $k(n) \leq$ $\frac{1-\epsilon}{2 \log |A|} \log n$ vale

$$
\sqrt{n} D\left(\hat{\nu}_{k(n), n} \| \nu_{k(n)}\right) \stackrel{\mathrm{P}}{\rightarrow} 0 .
$$


Prova. Como consequência das desigualdades de convexidade temos:

$$
\begin{aligned}
0 \leq D\left(\hat{\nu}_{k(n), n} \| \nu_{k(n)}\right) & \leq \log \left(\sum_{a_{1}^{k(n)}} \frac{\left(\hat{\nu}_{k(n), n}\left(a_{1}^{k(n)}\right)\right)^{2}}{\nu\left(a_{1}^{k(n)}\right)}\right) \\
& =\log \left(1+\sum_{a_{1}^{k(n)}} \frac{\left(\hat{\nu}_{k(n), n}\left(a_{1}^{k(n)}\right)-\nu\left(a_{1}^{k(n)}\right)\right)^{2}}{\nu\left(a_{1}^{k(n)}\right)}\right) \\
& \leq \sum_{a_{1}^{k(n)}} \frac{\left(\hat{\nu}_{k(n), n}\left(a_{1}^{k(n)}\right)-\nu\left(a_{1}^{k(n)}\right)\right)^{2}}{\nu\left(a_{1}^{k(n)}\right)} \\
& =\frac{1}{n-k(n)+1}\left[\sum_{a_{1}^{k(n)}}\left(\frac{\sum_{i=1}^{n-k(n)+1}\left(1\left(x_{i}^{i+k-1}=a_{1}^{k(n)}\right)-\nu\left(a_{1}^{k(n)}\right)\right)}{\sqrt{(n-k(n)+1) \nu\left(a_{1}^{k(n)}\right)}}\right)^{2}\right] .
\end{aligned}
$$

Usando (2.3.4) e o Lemma 4.3.4, temos

$$
\begin{aligned}
\mathbf{E}_{\nu}\left(\sqrt{n} D\left(\hat{\nu}_{k(n), n} \| \nu_{k(n)}\right)\right) & =O\left(\frac{1}{\sqrt{n}}\right) \sum_{a_{1}^{k(n)}} \frac{\left(\sigma^{2}\right)_{I\left[a_{1^{k(n)}}\right]}^{n}}{\nu\left(\left[a_{1^{k(n)}}\right]\right)} \\
& =O\left(\frac{1}{\sqrt{n}}\right)|A|^{k(n)} \\
& =O\left(n^{-\frac{\epsilon}{2}}\right) .
\end{aligned}
$$

Usando a desigualdade de Chebychev podemos concluir a prova.

\subsection{Teorema-limite central para a entropia empírica}

Consideraremos $\left(X_{n}\right)_{n \in \mathbb{Z}}$ uma c.o.i. positiva e log-contínua com decaimentos exponenciais.

Teorema 4.6.1. Seja $\epsilon>0$. Para qualquer sequência $(k(n))_{n \in \mathbb{N}}$ tal que $k(n) \leq$ $\frac{1-\epsilon}{2 \log |\mathcal{A}|} \log n$, vale 
- $S e \sigma_{\phi}^{2}>0$

$$
\lim _{n \rightarrow \infty} \mathrm{P}\left(\sqrt{\frac{n}{\sigma_{\phi}^{2}}}\left(\hat{h}_{k(n), n}-h_{k}\right)<\lambda\right)=\frac{1}{\sqrt{2 \pi}} \int_{-\infty}^{\lambda} e^{-u^{2} / 2} d u .
$$

uniformemente com respeito a $\lambda$;

- Se $\sigma_{\phi}^{2}=0$ então

$$
\sqrt{n}\left(\hat{h}_{k(n), n}-h_{k}(n)\right) \stackrel{\mathrm{P}}{\rightarrow} 0 .
$$

Prova. Escreveremos

$$
\begin{aligned}
y_{k(n), n} & :=\hat{h}_{k(n), n}-h_{k}(n) \\
& =\xi_{k(n), n}+\lambda_{k(n), n}
\end{aligned}
$$

onde

$$
\begin{aligned}
\xi_{k(n), n} & :=\sum_{a_{-k(n)}^{0}} \hat{\nu}_{k(n)+1, n}\left(a_{-k(n)}^{0}\right) \log \frac{\nu_{k(n)+1}\left(a_{0} \mid a_{-k(n)}^{-1}\right)}{\hat{\nu}_{k(n)+1, n}\left(a_{0} \mid a_{-k(n)}^{-1}\right)} \\
& =-D\left(\hat{\nu}_{k(n)+1, n} \| \nu_{k(n)+1}\right)+D\left(\hat{\nu}_{k(n), n} \| \nu_{k(n)}\right) .
\end{aligned}
$$

$\mathrm{e}$

$$
\begin{aligned}
\lambda_{k(n), n}:= & -\sum_{a_{-k(n)}^{0}} \hat{\nu}_{k(n)+1, n}\left(a_{-k(n)}^{0}\right) \log \left(\nu_{k(n)+1}\left(a_{0} \mid a_{-k(n)}^{-1}\right)\right) \\
& +\sum_{a_{-k(n)}^{0}} \nu_{k(n)+1}\left(a_{-k(n)}^{0}\right) \log \left(\nu_{k(n)+1}\left(a_{0} \mid a_{-k(n)}^{-1}\right)\right) . \\
= & \frac{1}{n-k+1} \sum_{i=1}^{n-k+1}\left(T_{i} \phi_{k(n)}-\mathbf{E}_{\nu}\left[\phi_{k(n)}\right]\right) \\
= & \frac{1}{n} s_{k(n), n}\left(\phi_{k(n)}\right)+O\left(\frac{k(n)}{n}\right)
\end{aligned}
$$

onde $s_{k(n), n}$ foi definido em (4.1.8).

Como consêquencia do Teorema 4.5.1 temos que, $\sqrt{n} \xi_{k(n), n}$ converge em probabilidade para zero.

Com consequência das Lemas 4.3.1, 4.3.3, 4.3.5 podemos concluir que as funções $\phi_{k}$, satisfazem as condições do Teorema 4.1.1. Então as variáveis aleatórias $\sqrt{n} \lambda_{k(n), n}$ tem flutuações gaussianas. Isto conclui a prova do teorema. 
Teorema 4.6.2. Seja $\epsilon>0$. Para qualquer sequência $(k(n))_{n \in \mathbb{N}}$, tal que $k(n) \leq$ $\frac{1-\epsilon}{2 \log |\mathcal{A}|} \log n$, vale

- Se $\sigma_{\phi}^{2}>0$

$$
\lim _{n \rightarrow \infty} \mathrm{P}\left(\sqrt{\frac{n}{\sigma_{\phi}^{2}}}\left(\frac{1}{k(n)} \hat{H}_{k(n), n}-\frac{1}{k(n)} H_{k}(n)\right)<\lambda\right)=\frac{1}{\sqrt{2 \pi}} \int_{-\infty}^{\lambda} e^{-u^{2} / 2} d u
$$

uniformemente com respeito a $\lambda$;

- Se $\sigma_{\phi}^{2}=0$ então

$$
\sqrt{n}\left(\frac{1}{k(n)} \hat{H}_{k(n), n}-\frac{1}{k(n)} H_{k}(n)\right) \stackrel{\mathrm{P}}{\rightarrow} 0 .
$$

Prova. Escreveremos

$$
\begin{aligned}
Y_{k(n), n} & :=\frac{1}{k(n)}\left(\hat{H}_{k(n), n}-H_{k(n)}\right) \\
& =D\left(\hat{\nu}_{k(n), n} \| \nu_{k(n)}\right)+\Lambda_{k(n), n} .
\end{aligned}
$$

onde

$$
\Lambda_{k(n), n}=\frac{\sum_{i=0}^{n-k(n)}\left(T_{i} e_{k(n)}-\mathbf{E}_{\nu}\left[e_{k(n)}\right]\right)}{n-k(n)+1} .
$$

Como consêquencia do Teorema 4.5.1 que $\sqrt{n} D\left(\hat{\nu}_{k(n), n} \| \nu_{k(n)}\right)$ converge em probabilidade para zero.

De outro lado, em consequência das Lemas 4.3.1, 4.3.3, 4.3.5 podemos concluir que as funções $e_{k(n)}$, satisfazem as condições do Teorema 4.1.1. Então as variáveis aleatórias $\sqrt{n} \Lambda_{k(n), n}$ tem flutuações gaussianas. Isto conclui a prova do teorema.

\subsubsection{Velocidade de convergência}

Lema 4.6.3. Seja $X=\left(X_{n}\right)_{n \in Z}$ uma c.o.i. positiva com taxa de log-continuidadenuidade $\gamma_{k}$. Para qualquer $k \geq 0$ temos

$$
0 \leq h_{k}-h \leq \gamma_{k}
$$


$\operatorname{Se} \sum_{i=0}^{\infty} \gamma_{i}<+\infty$ então

$$
\frac{1}{k} H_{k}-h=O\left(\frac{1}{k}\right) .
$$

Prova. Usando as desigualdages de convexidade temos: $0 \leq d_{k} \leq d_{k-1}$.

$$
\begin{aligned}
d_{k} & :=h_{k}-h \\
& =\int_{a_{-\infty}^{0}} d \nu\left(a_{-\infty}^{0}\right) \log \frac{\nu\left(a_{0} \mid a_{-\infty}^{-1}\right)}{\nu\left(a_{0} \mid a_{-k}^{-1}\right)} \\
& \leq \int_{a_{-\infty}^{0}} d \nu\left(a_{-\infty}^{0}\right)\left(\frac{\nu\left(a_{0} \mid a_{-\infty}^{-1}\right)}{\nu\left(a_{0} \mid a_{-k}^{-1}\right)}-1\right) \\
& \leq \gamma_{k} .
\end{aligned}
$$

Como $H_{k}=\sum_{i=1}^{k} h_{k}$ temos $\frac{1}{k} H_{k}-h=\frac{1}{k} \sum_{i=0}^{k} d_{i}$.

\subsubsection{Flutuações dos dois estimadores da entropia em torno da entropia do processo}

Podemos finalmente, nesta Seção, apresentar os dois teoremas centrais desta tese. Os dois respondem à pergunta deixada em aberto no artigo de Iosifescu (1965), á saber, como flutuam as entropias empíricas em torno da entropia do processo, no caso de cadeias de ordem infinita. O Teorema 4.6.4 mostra que as flutuações da $k(n)$-ésima entropia empírica condicionada em torno da entropia do processo são normais com variância finita. O Teorema 4.6 .5 mostra que, com qualquer normalização, o outro estimador considerado nesta tese, a $k(n)$-ésima entropia empírica, não tem flutuações normais em torno da entropia. Esta é uma diferença importante entre o caso Markoviano e cadeias de ordem infinita.

Consideraremos $\left(X_{n}\right)_{n \in \mathbb{Z}}$ uma c.o.i. positiva e log-contínua com decaimentos exponenciais, isto é $\gamma_{m}=e^{-c m} \operatorname{com} c>\log |\mathcal{A}|$.

Com consequência do teorema (4.6.1) temos o seguinte Teorema.

Teorema 4.6.4. Seja $\epsilon>0$. Para qualquer sequência $\frac{1+\varepsilon}{2 c} \log n \leq(k(n))_{n \in \mathbb{N}}$, tal que $k(n) \leq \frac{1-\epsilon}{2 \log |\mathcal{A}|} \log n$, vale

- $\operatorname{Se} \sigma_{\phi}^{2}>0$

$$
\lim _{n \rightarrow \infty} \mathrm{P}\left(\sqrt{\frac{n}{\sigma_{\phi}^{2}}}\left(\hat{h}_{k(n), n}-h\right)<\lambda\right)=\frac{1}{\sqrt{2 \pi}} \int_{-\infty}^{\lambda} e^{-u^{2} / 2} d u .
$$


uniformemente com respeito a $\lambda$;

- Se $\sigma_{\phi}^{2}=0$ então

$$
\sqrt{n}\left(\hat{h}_{k(n), n}-h\right) \stackrel{\mathbf{P}}{\rightarrow} 0 .
$$

Prova.

$$
\sqrt{n}\left(\hat{h}_{k(n), n}-h\right)=\sqrt{n}\left(\hat{h}_{k(n), n}-h_{k(n)}\right)+\sqrt{n} d_{k(n)} .
$$

Usando (4.6.7) temos

$$
\sqrt{n} d_{k(n)} \leq \sqrt{n} \gamma_{k(n)} \leq n^{-\frac{\epsilon}{2}}
$$

Em consequência do Teorema 4.6.1 e de (4.6.11) podemos concluir a prova.

Teorema 4.6.5. Para qualquer sequência $(k(n))_{n \in \mathbb{N}}$, tal que $k(n) \leq \frac{1-\epsilon}{2 \log |\mathcal{A}|} \log n$, vale

1) $S e \lim _{n \rightarrow \infty} \frac{q(n)}{k(n)}=0$ então

$$
q(n)\left(\left(\frac{1}{k(n)} \hat{H}_{k(n), n}-h\right) \stackrel{\mathbf{P}}{\rightarrow} 0 .\right.
$$

2) Se $\lim _{n \rightarrow \infty} \frac{q(n)}{k(n)}=\alpha$ onde $0<\alpha<+\infty$, então

$$
q(n)\left(\left(\frac{1}{k(n)} \hat{H}_{k(n), n}-h\right) \stackrel{\mathrm{P}}{\rightarrow} \alpha \sum_{i=0}^{\infty} d_{i} .\right.
$$

3) Se $\lim _{n \rightarrow \infty} \frac{q(n)}{k(n)}=+\infty$ então para qualquer $\lambda \in \mathbb{R}$ vale

$$
\lim _{n \rightarrow \infty} \mathrm{P}\left(q(n)\left(\frac{1}{k(n)} \hat{H}_{k(n), n}-h\right)>\lambda\right)=1 .
$$

Prova.

$$
\begin{aligned}
q(n)\left(\frac{1}{k(n)} \hat{H}_{k(n), n}-h\right) & =q(n)\left(Y_{k(n), n}+D_{k(n)}\right) \\
& =\frac{q(n)}{\sqrt{n}}\left(\sqrt{n} Y_{k(n), n}\right)+q(n) D_{k(n)} .
\end{aligned}
$$


onde

$$
Y_{k(n), n}:=\frac{1}{k(n)} \hat{H}_{k(n), n}-\frac{1}{k(n)} H_{k(n)} .
$$

e

$$
D_{k(n)}:=\frac{1}{k(n)} H_{k(n)}-h .
$$

Se $\lim _{n \rightarrow \infty} \frac{q(n)}{k(n)}=\alpha$, usando o Teorema 4.6.2 temos

$$
\lim _{n \rightarrow \infty} q(n) Y_{k(n), n}=0 .
$$

De outro lado usando (4.6.8) temos

$$
\begin{aligned}
q(n) D_{k(n)} & =q(n)\left(\frac{1}{k(n)} H_{k(n), n}-h\right) \\
& =O\left(\frac{q(n)}{k(n)}\right) .
\end{aligned}
$$

Isto conclui a prova. 


\section{Referências Bibliográficas}

[1] K. ATHREYA and P. NEY (1978). A new approach to the limit theory of recurrent Markov chain. Trans. Am. Math. Soc. 245, 493-501.

[2] H. BERBEE (1987). Chains with infinite connections: Uniqueness and Markov representation. Probab. Th. Rel. Fields. 76, 243-253.

[3] P. BLLINGSLEY (1995). Probability and Measures. Third edition. Wiley Series in Probability and Mathematical Statistics. A Wiley-Interscience Publication. John Wiley \& Sons, Inc., New York. xiv+593 pp.

[4] X. BRESSAUD, R. FERNÁNDEZ, and A. GALVES (1999a). Speed of $\bar{d}$-convergence for Markov approximations of chains with complete connections. A coupling approach. Stoc. Process. Appl. 83, 127-138.

[5] X. BRESSAUD, R. FERNÁNDEZ, and A. GALVES (1999b). Decay of Correlation for non Holderian Dynamics. A coupling Approach. El. Jour. Prob. 4, No 3, 1-19.

[6] F. COMETS, R. FERNÁNDEZ, P.A. FERRARI (2000). Processes with Long Memory: Construction, Perfect Simulation and Regeneration. Preprint. (http://arXiv.org/ Math.PR 0009204) or (http://www.ime.usp.br/ pablo/publications.html. file N 71)

[7] T. COVER and J.A. THOMAS (1991). Elements of information theory. Wiley Series in Telecommunications. A Wiley-Interscience Publication. John Wiley \& Sons, Inc., New York. xxiv+542 pp.

[8] I. CSISZÁR (1989). Lecture Notes. Univ. Maryland. 32 pp.

[9] I. CSISZÁR (2000). Large scale typicality of Markov sample paths and consistency of MLD order estimators. Preprint $24 \mathrm{pp}$. 
[10] I. CSISZÁR and P.C. SHIELDS (2000). The consistency of the BIC Markov order estimator. Ann. Statist. 28, no. 6, 1601-1619.

[11] W. DOEBLIN and R. FORTET (1937). Sur les chaînes à liaisons complètes. Bull. Soc. Math. France. 65, 132-148.

[12] R. FERNÁNDEZ, P.A. FERRARI and A. GALVES (2001). Coupling, renewal and perfect simulation of chains of infinite order. Notes for the V Brazilian School of Probability, Ubatuba, August 2001 or (http://www.ime.usp.br/ pablo/publications.html. file N 78)

[13] P.A. FERRARI, A. MAASS, S. MARTÍNEZ and P. NEY (2000). Cesàro mean distribution of group automata starting from measures with summable decay. Ergodic Theory Dynam. Systems. 20, No. 6, 1657-1670.

[14] R.M. GRAY (1990). Entropy and information theory. Springer-Verlag, New York. xxiv +332 pp.

[15] G.R. GRIMMETT and D.R. STIRZAKER (1992). Probability and random processes. Second edition. The Clarendon Press, Oxford University Press, New York, 1992. xii+541 pp.

[16] T. E. HARRIS (1955). On chains of infinite order. Pacific J. Math. 5:707724.

[17] M. IOSIFESCU (1961). On the asymptotic behavior of chains with complete connections. Comunicãrile Acad. RPR. 11, 619-24.

[18] M. IOSIFESCU (1965). Sampling Entropy for Random Homogeneus Systems with Complete Connections. Ann. Math. Stat. 36, 1433-1436.

[19] M. IOSIFESCU and S. GRIGORESCU (1990). Dependence with complete connections and its applications. Cambridge Tracts in Mathematics, 96. Cambridge University Press, Cambridge, 1990. xiv+304 pp.

[20] S.P. LALLEY (1986). Regeneration representation for one-dimensional Gibbs states. Ann. Prob. 14, 1262-1271.

[21] K. MARTON and P.C. SHIELDS (1994). Entropy and the consistent estimation of joint distributions. Ann. Prob. 22, No 2, 960-977. 
[22] K. MARTON and P.C. SHIELDS (1996). Correction: "Entropy and the consistent estimation of joint distributions" [Ann. Probab. 22 (1994), no. 2, 960-977]. Ann. Prob. 24, No 1, 541-545.

[23] G. MIHOC (1963). The limit law for sums of vector valued random variables forming a multiple stationary chain with complete connections. em Comunicãrile Acad. RPR., 13, 5-9.

[24] E. NUMMELIN (1978). A splitting technique for Harris recurent Markov chains. Z. Wahrsch. Verw. Gebiete. 43, 309-318.

[25] O. ONICESCU and G. MIHOC (1935). Sur les chaînes de variables statistiques. Bull Sci. Math. 59, 174-92.

[26] O. ONICESCU and G. MIHOC (1940). Le comportement asymptotique des chaînes à liaisons complètes. Disq. Math. Phys. 1, 61-2.

[27] D.S. ORNSTEIN, B. WEISS (1983). The Shannon-McMillan-Breiman theorem for a class of amenable groups. Israel J. Math. 44, No 1, 53-60.

[28] D. S. ORNSTEIN, B. WEISS (1990). How sampling reveals a process. Ann. Prob. 18, 905-930.

[29] P.C. SHIELDS (1996). The ergodic theory of discrete sample paths. Graduate Studies in Mathematics, 13. American Mathematical Society, Providence, RI. xii+249 pp.

[30] P. SHIELDS (2001). Private Communication. 